\title{
Existence of Random Attractors for a Class of Second-Order Lattice Dynamical Systems with Brownian Motions
}

\author{
Yamin Wang, ${ }^{1,2}$ Ziqiao Huang, ${ }^{1}$ Fuad E. Alsaadi, ${ }^{3}$ Stanislao Lauria, ${ }^{4}$ and Yurong Liu ${ }^{1,3}$ \\ ${ }^{1}$ Department of Mathematics, Yangzhou University, Yangzhou 225002, China \\ ${ }^{2}$ Department of Basis Course, Lianyungang Technical College, Lianyungang 222006, China \\ ${ }^{3}$ Department of Electrical and Computer Engineering, Faculty of Engineering, King Abdulaziz University, Jeddah 21589, Saudi Arabia \\ ${ }^{4}$ Department of Computer Science, Brunel University, Uxbridge, Middlesex UB8 3PH, UK
}

Correspondence should be addressed to Yurong Liu; liuyurong@gmail.com

Received 25 September 2014; Accepted 2 November 2014; Published 3 December 2014

Academic Editor: Bo Shen

Copyright (C) 2014 Yamin Wang et al. This is an open access article distributed under the Creative Commons Attribution License, which permits unrestricted use, distribution, and reproduction in any medium, provided the original work is properly cited.

\begin{abstract}
This paper is concerned with the random attractors for a class of second-order stochastic lattice dynamical systems. We first prove the uniqueness and existence of the solutions of second-order stochastic lattice dynamical systems in the space $F=l_{\lambda}^{2} \times l^{2}$. Then, by proving the asymptotic compactness of the random dynamical systems, we establish the existence of the global random attractor. The system under consideration is quite general, and many existing results can be regarded as the special case of our results.
\end{abstract}

\section{Introduction}

We consider the following second-order stochastic lattice dynamical system:

$$
\begin{array}{r}
\ddot{u}+\xi A \dot{u}+h(\dot{u})+A u+\eta u+f(u)=g+\dot{W}(t), \\
t>0, \\
u(0)=\left(u_{i, 0}\right)_{i \in \mathbb{Z}^{n}}=u_{0}, \quad \dot{u}(0)=\left(u_{1 i, 0}\right)_{i \in \mathbb{Z}^{n}}=u_{10},
\end{array}
$$

where $u=\left(u_{i}\right)_{i \in \mathbb{Z}^{n}} \in l^{2}, \dot{u}=\left(\dot{u}_{i}\right)_{i \in \mathbb{Z}^{n}} \in l^{2}$ are real-value functions on $\mathbb{R}^{+} ; \xi=\left(\xi_{i}\right)_{i \in \mathbb{Z}^{n}}$ and $\eta=\left(\eta_{i}\right)_{i \in \mathbb{Z}^{n}}$ are given vectors satisfying bounded conditions; $g=\left(g_{i}\right)_{i \in \mathbb{Z}^{n}} \in l^{2}$; $f(u)=\left(f_{i}\left(u_{i}\right)\right)_{i \in \mathbb{Z}^{n}}$ and $h(\dot{u})=\left(h_{i}\left(\dot{u}_{i}\right)\right)_{i \in \mathbb{Z}^{n}}$ are nonlinear terms satisfying some growth assumptions to be given later; $A$ is the linear operator on $l^{2}$. In (1), $W(t)=W(t, \omega)=$ $\sum_{i \in \mathbb{Z}^{n}} a_{i} w_{i}(t, \omega) e_{i}$, where $a=\left(a_{i}\right)_{i \in \mathbb{Z}^{n}} \in l^{2}$ and $e_{i} \in l^{2}$ denotes the element having 1 at position $i$ and all the other components 0 and $\left\{w_{i}, i \in \mathbb{Z}^{n}\right\}$ are independent two-side Brownian motions.

Lattice dynamical systems (LDSs) are infinite systems of ordinary differential equations, modeled on an underlying spatial lattice with some regular structure, for example, the integer lattice in the plane. Such systems arise as models in many applications, including image processing and pattern recognition, electrical engineering laser systems, biology, and material science; see $[1-8]$ and the references therein. LDSs in one sense lie between ordinary and partial differential equations, but very often they exhibit new phenomena not found in either of these fields. LDSs raise a host of challenges to the researcher and are of broad interest to scientists and mathematicians.

So far, various properties of solutions about LDSs have been studied by many authors, such as the traveling solutions, the chaotic properties of solutions, and the phenomena of synchronization (see, e.g., $[4,5,9])$. One of the most important problems in mathematical physics is understanding of the asymptotic behavior of dynamical systems. Global attractor theory is an important tool to study the asymptotic behavior of infinite dimensional systems. For dissipative infinite dimensional dynamical systems given by partial differential equations, global attractor theory has been well developed; see $[10,11]$ and references therein. Recently, the long-term behavior of LDS has gained the extensive attention. For the LDSs without noise, the first result on existence of global attractors was established by Bates et al. in [12]. Since then, much work has been done for either first-order or second-order deterministic LDS (see, e.g., [12, 13]).

On the other hand, when modeling real world systems, stochastic disturbance is probably one of the main resources 
of the performance degradations of the dynamical systems, since the actual dynamic behavior is very often a noisy process brought on by random fluctuations from probabilistic causes. Stochastic systems have found successful applications in more and more branches of science and engineering. Random attraction as an interesting dynamic behavior has received increasing research attention. For stochastic partial differential equations, Ruelle has initiated the study of global random attractors in [14]. And the fundamental theory of global attractors for stochastic partial differential equations has been established and developed by Crauel, Debussche, Flandoli, Schmalfuss, and others; see, for example, [15-18] and the references therein. Very recently, much attention has been focused on lattice dynamical system with stochastic noises. Bates et al. [19] first studied the existence of global random attractor for a class of first-order dynamical systems driven by white noises on lattice $\mathbb{Z}$. Then, Lv and Sun [20] have extended the result in [19] to generalized first-order stochastic systems on the lattice $\mathbb{Z}^{k}$. For some latest results on first-order random attractors, we refer readers to, for example, [21] and the references therein.

For the second-order SLDS with stochastic noises on the lattice $\mathbb{Z}$ or $\mathbb{Z}^{k}$, the existence of the random attractor is receiving the attention from research community $[22,23]$. For example, [22] investigated the asymptotic behavior for a class of second-order stochastic lattice dynamical systems and proved the existence of the random attractor for the concerned second-order SLDS. Paper [23] addressed the asymptotic behavior of solutions to second-order SLDS with random coupled coefficients and multiplicative white noises in weighted spaces of infinite sequences and discussed the existence of a tempered random bounded absorbing set and a random attractor for the SLDS. However, the asymptotic behavior of second-order SLDS has not yet been fully investigated because of the technical complexity and remains open and challenging. In this paper, based on the idea of $[13,19]$, we aim to prove the existence of a global random attractor for a class of second-order SLDS (1). It is worth pointing out that the second-order SLDS considered in this paper is quite general, and many existing results can be viewed as the special cases of our results.

This paper is organized as follows. In Section 2, we introduce some basic concepts related to stochastic dynamical systems and the global random attractor. Meanwhile, we present some notations and give a simple description of our system. In Section 3, Some bounded conditions and assumptions of nonlinear terms are given, and the existence and uniqueness of solutions of system (1) are established. In Section 4, we prove the existence of an absorbing set. In Section 5, we establish the existence conditions for a global random attractor of system (1), and some concluding remarks are given in Section 6.

\section{Preliminaries and Equivalent Norm}

2.1. Preliminaries. In this subsection, we recall some basic concepts about random dynamical systems and the definition of random global attractor (see $[17,19,24]$ for details).
Let $\left(H,\|\cdot\|_{H}\right)$ be a Hilbert space and $(\Lambda, \mathscr{F}, \mathbb{P})$ a probability space. Denote $\mathscr{D}$ as a collection of random subsets of $H$. A continuous random dynamical system $(s(t, \omega))$ over $\left(\Lambda, \mathscr{F}, \mathbb{P},\left(\theta_{t}\right)_{t \in \mathbb{R}}\right)$ is defined as follows.

Definition 1. A stochastic process $(s(t))_{t \geq 0}$ is a continuous random dynamical system over $\left(\Lambda, \mathscr{F}, \mathbb{P},\left(\theta_{t}\right)_{t \in \mathbb{R}}\right)$ if $s(t)$ is $(\mathscr{B}[0, \infty) \times \mathscr{F} \times \mathscr{B}(H), \mathscr{B}(H))$-measurable and, for all $\omega \in \Lambda$,

(S1) the mapping $s(\cdot, \omega)(\cdot):[0, \infty] \times H \rightarrow H$ is continuous;

(S2) $s(0, \omega)(\cdot)$ is the identity operator on $H$;

(S3) $s(p+t, \omega)(\cdot)=s\left(t, \theta_{p} \omega\right) \circ s(p, \omega)(\cdot)$ for all $p, t \geq 0$ (cocycle property).

Definition 2. A random bounded set $B(\omega) \subset H$ is called tempered with respect to $\left(\theta_{t}\right)_{t \in \mathbb{R}}$, if, for all $\omega \in \Lambda$,

$$
\lim _{t \rightarrow \infty} e^{-\beta t} d\left(B\left(\theta_{t} \omega\right)\right)=0, \quad \forall \beta>0,
$$

where $d(B)=\sup _{x \in B}\|x\|_{H}$.

Definition 3. A random set $K$ is called an absorbing set in $\mathscr{D}$ if, for all $B \in \mathscr{D}$ and a.e. $\omega \in \Lambda$, there exists $t_{B}(\omega)>0$ such that

$$
s\left(t, \theta_{-t} \omega\right)\left(B\left(\theta_{-t} \omega\right)\right) \subset K(\omega), \quad \forall t \geq t_{B}(\omega) .
$$

Definition 4. A random set $\mathscr{A}$ is called a global random (D) attractor for $s(t)$ if the following hold:

(A1) $\mathscr{A}$ is a random compact set; that is, $\omega \mapsto d(x, \mathscr{A}(\omega))$ is measurable for every $x \in H$, and $\mathscr{A}(\omega)$ is compact for a.e. $\omega \in \Lambda$;

(A2) $\mathscr{A}$ is a strictly invariant set;

(A3) $\mathscr{A}$ attracts all sets in $\mathscr{D}$; that is, for all $B \in \mathscr{D}$ and a.e. $\omega \in \Lambda$ one has

$$
\lim _{t \rightarrow \infty} d\left(s\left(t, \theta_{-t} \omega\right)\left(B\left(\theta_{-t} \omega\right)\right), \mathscr{A}(\omega)\right)=0,
$$

where $d(A, B)=\sup _{x \in A} \inf _{y \in B}\|x-y\|_{H}$ is Hausdorff semimetric (for any $A \subseteq H, B \subseteq H$ ). Also, the collection $\mathscr{D}$ is called the domain of attraction of $\mathscr{A}$.

The following theorem is needed to prove the existence of global random attractor of system (1).

Theorem 5. Let $K \in \mathscr{D}$ be an absorbing set for the continuous random dynamical system $(s(t))_{t \geq 0}$. Suppose the set $K$ is closed and, for a.e. $\omega \in \Lambda$, $K$ satisfies the following asymptotic compactness condition: each sequence $\phi_{n} \in s\left(t_{n}, \theta_{-t_{n}}\right)\left(K\left(\theta_{-t_{n}} \omega\right)\right)$ (when $t \rightarrow \infty$ ) has a convergent subsequence in $H$. Then, $s(t, \omega)$ has a unique global random attractor

$$
\mathscr{A}(\omega)=\bigcap_{\tau \geq t_{K}(\omega) t \geq \tau} \overline{\bigcup_{s}\left(t, \theta_{-t} \omega\right)\left(K\left(\theta_{-t} \omega\right)\right)} .
$$

Proof. The proof of this theorem is similar to [12], so it is omitted here. 


\subsection{Equivalent Norm. Let}

$$
\begin{aligned}
l^{2}=\left\{u=\left(u_{i}\right)_{i \in \mathbb{Z}^{n}} \mid \sum_{i \in \mathbb{Z}^{n}} u_{i}^{2}<+\infty,\right. \\
\left.u_{i} \in R, i=\left(i_{1}, i_{2}, \ldots, i_{n}\right) \in \mathbb{Z}^{n}\right\} .
\end{aligned}
$$

For all $u, v \in l^{2}$, we define the inner product $(\cdot, \cdot)$ and norm $\|\cdot\|$ as follows:

$$
(u, v)=\sum_{i \in \mathbb{Z}^{n}} u_{i} v_{i}, \quad\|u\|^{2}=(u, u)=\sum_{i \in \mathbb{Z}^{n}} u_{i}^{2}
$$

In this paper, we assume that the linear operator $A$ in (1) can be decomposed as

$$
A=A_{1}+A_{2}+\cdots+A_{n}, \quad A_{j}=D_{j} D_{j}^{*}, \quad j=1,2, \ldots, n,
$$

where $D_{j}$ and $D_{j}^{*}: l^{2} \rightarrow l^{2}$ are bounded linear operators defined by

$$
\begin{aligned}
& \left(D_{j} u\right)_{i}=u_{\left(i_{1}, \ldots, i_{j}+1, \ldots, i_{n}\right)}-u_{\left(i_{1}, \ldots, i_{j}, \ldots, i_{n}\right)}, \\
& \left(D_{j}^{*} u\right)_{i}=u_{\left(i_{1}, \ldots, i_{j}-1, \ldots, i_{n}\right)}-u_{\left(i_{1}, \ldots, i_{j}, \ldots, i_{n}\right)} .
\end{aligned}
$$

It follows readily that

$$
\left(A_{j} u\right)_{i}=2 u_{\left(i_{1}, \ldots, i_{j}, \ldots, i_{n}\right)}-u_{\left(i_{1}, \ldots, i_{j}-1, \ldots, i_{n}\right)}-u_{\left(i_{1}, \ldots, i_{j}+1, \ldots, i_{n}\right)} .
$$

For all $u=\left(u_{i}\right)_{i \in \mathbb{Z}^{n}}, v=\left(v_{i}\right)_{i \in \mathbb{Z}^{n}} \in l^{2}$, define the bilinear forms by

$$
\begin{aligned}
& (u, v)_{\lambda}=\gamma \sum_{j=1}^{n}\left(D_{j} u, D_{j} v\right)+\lambda(u, v), \\
& \|u\|_{\lambda}^{2}=\gamma \sum_{j=1}^{n}\left|D_{j} u\right|^{2}+\lambda\|u\|^{2},
\end{aligned}
$$

where $\gamma=1-\varepsilon \overline{\bar{\xi}}$ ( $\varepsilon$ and $\overline{\bar{\xi}}$ are given constants).

Now, we prove that the spaces $l^{2}=\left(l^{2},(\cdot, \cdot),\|\cdot\|\right)$ and $l_{\lambda}^{2}=$ $\left(l_{\lambda}^{2},(\cdot, \cdot)_{\lambda},\|\cdot\|_{\lambda}\right)$ are equivalent spaces.

Lemma 6. The two bilinear forms $(\cdot, \cdot),(\cdot, \cdot)_{\lambda}$ in (7) and (11) are both the inner products, and the resulting norms $\|\cdot\|$ in (7) and $\|\cdot\|_{\lambda}$ in (11) are equivalent.
Proof. It is easy to check that the two bilinear forms $(\cdot, \cdot)$ and $(\cdot, \cdot)_{\lambda}$ are both the inner products. We now only need to show that the norms $\|\cdot\|$ and $\|\cdot\|_{\lambda}$ are equivalent. Noticing that

$$
\begin{gathered}
\lambda\|u\|^{2} \leq\|u\|_{\lambda}^{2}=\gamma \sum_{j=1}^{n}\left|D_{j} u\right|^{2}+\lambda\|u\|^{2}, \\
\|u\|_{\lambda}^{2}=\gamma \sum_{j=1}^{n}\left|D_{j} u\right|^{2}+\lambda\|u\|^{2} \\
\leq \gamma \sum_{j=1}^{n} \sum_{i \in \mathbb{Z}^{n}}\left|u_{\left(i_{1}, \ldots, i_{j}+1, \ldots, i_{n}\right)}-u_{\left(i_{1}, \ldots, i_{j}, \ldots, i_{n}\right)}\right|^{2}+\lambda\|u\|^{2} \\
\leq(4 n \gamma+\lambda)\|u\|^{2},
\end{gathered}
$$

it follows that the norms $\|\cdot\|$ and $\|\cdot\|_{\lambda}$ are equivalent. The proof is completed.

Let $F=l_{\lambda}^{2} \times l^{2}$, where $l_{\lambda}^{2}:=\left(l_{\lambda}^{2},(\cdot, \cdot)_{\lambda},\|\cdot\|_{\lambda}\right)$. From Lemma 6, we know that $F=l_{\lambda}^{2} \times l^{2}$ is a Hilbert space.

\section{Existence and Uniqueness of Solutions}

In this section, we will deal with the existence and uniqueness of solutions of system (1). For system (1), we make the following assumption (bounded conditions).

(C1):

$$
\begin{aligned}
0<\bar{\xi} \leq \xi_{i} \leq \overline{\bar{\xi}}<+\infty, & 0<\lambda \leq \eta_{i} \leq \overline{\bar{\eta}}<+\infty, \\
& i=\left(i_{1}, i_{2}, \ldots, i_{n}\right) \in \mathbb{Z}^{n},
\end{aligned}
$$

where $\lambda, \overline{\bar{\eta}}, \bar{\xi}$, and $\overline{\bar{\xi}}$ are known positive constants;

(C2): For all $i \in \mathbb{Z}^{n}, f_{i}(s) \in C^{1}(\mathbb{R})$ and for each of bounded sets $B \sup _{s \in B}\left|f_{i}^{\prime}(s)\right|<+\infty$; moreover,

$$
\begin{gathered}
f_{i}(s) s \geq c_{1} V_{i}(s) \geq c_{2}|s|^{2 p+2} \geq 0, \quad \forall s \in \mathbb{R}, \quad i \in \mathbb{Z}^{n}, \\
\left|f_{i}(s)\right| \leq c_{2}\left(|s|^{2 p+1}+|s|\right), \quad \forall s \in \mathbb{R}, \quad i \in \mathbb{Z}^{n},
\end{gathered}
$$

where $c_{1}, c_{2}$, and $p$ are positive constants and $V_{i}(s)=$ $\int_{0}^{s} f_{i}(t) d t$

(C3): Assume $h_{i} \in C^{1}((\mathbb{R}, \Lambda), \mathbb{R})$. For all $\omega \in \Lambda, h_{i}(0, \omega)=$ 0 . Furthermore, there exist constants $\alpha$ and $\beta$ such that

$$
0<\alpha \leq h_{i}^{\prime}(x, \omega) \leq \beta<+\infty, \quad \forall x \in \mathbb{R}, \omega \in \Lambda, i \in \mathbb{Z}^{n} .
$$

Also, $h_{i}$ satisfies cocycle property

$$
\begin{aligned}
& h_{i}(t+\tau, \omega)=h_{i}\left(t, \theta_{\tau} \omega\right)+h_{i}(\tau, \omega), \\
& \forall \tau, t \geq 0, \quad \omega \in \Lambda, \quad i \in \mathbb{Z}^{n} ;
\end{aligned}
$$

(C4):

$$
g=\left(g_{i}\right)_{i \in \mathbb{Z}^{n}} \in l^{2}
$$


(C5): $W(t)$ is a Brownian motion with values in $l^{2}$ defined on the probability space $(\Lambda, \mathscr{F}, \mathbb{P})$, where $\Lambda=\{\omega \epsilon$ $\left.C\left(\mathbb{R}, l^{2}\right): \omega(0)=0\right\}, \mathscr{F}$ is a complete $\sigma$-algebra, and $\mathbb{P}$ is the corresponding Wiener measure on $\mathscr{F}$. To be specific, $W(t)=W(t, \omega)=\sum_{i \in \mathbb{Z}^{n}} a_{i} w_{i}(t, \omega) e_{i}$, where $a=\left(a_{i}\right)_{i \in \mathbb{Z}^{n}} \in l^{2}$ and $e_{i} \in l^{2}$ denotes the element being 1 at position $i$ and all the other components being 0 and $\left\{w_{i}, i \in \mathbb{Z}^{n}\right\}$ are independent of the two-side Brownian motions.

Remark 7. As pointed out in [19], the function $f(s)=|u|^{2 p} u$ satisfies condition (C2).

Next, we show the existence and uniqueness of the solution to system (1) under assumptions (C1)-(C5).

Let $\varphi=(u, v)^{T}$ where $v=\dot{u}+\epsilon u-W, u \in l^{2}$ and $\epsilon=$ $\alpha \lambda /\left(\beta^{2}+4 \lambda\right)$, and $(4 p+2) /(4 p+3) \leq \epsilon \leq 1$. Equation (1) can be rewritten as the following equation with initial condition:

$$
\begin{gathered}
\dot{\varphi}=C(\varphi)+E(\varphi)+G(\varphi), \\
\varphi(0)=\left(u_{0}, u_{10}+\epsilon u_{0}\right)^{T},
\end{gathered}
$$

where

$$
\begin{gathered}
C(\varphi)=\left(\begin{array}{cc}
-\epsilon & 1 \\
\epsilon \xi A-A-\eta-\epsilon^{2} & -\xi A+\epsilon
\end{array}\right) \varphi, \\
G(\varphi)=\left(\begin{array}{c}
0 \\
-f(u)+g
\end{array}\right), \\
E(\varphi)=\left(\begin{array}{c}
W \\
(-\xi A+\epsilon) W-h(v-\epsilon u+W)
\end{array}\right) .
\end{gathered}
$$

Lemma 8. For all $\omega \in \Lambda$, if conditions (C1)-(C5) hold, then the operators $E$ and $G$ in (19) map $F=l_{\lambda}^{2} \times l^{2}$ into themselves, and they are locally Lipschitz on F.

Proof. From the above assumptions, it follows that

$$
\begin{aligned}
\|f(u)\|^{2} & =\|f(u)-f(0)\|^{2} \\
& =\sum_{i \in \mathbb{Z}^{n}}\left|f_{i}\left(u_{i}\right)-f_{i}(0)\right|^{2}=\sum_{i \in \mathbb{Z}^{n}}\left|f_{i}^{\prime}\left(\theta_{i} u_{i}\right)\right|^{2}\left|u_{i}\right|^{2} \\
& \leq\left(\sup _{r \in[-\|u\|,\|u\|]}\left|f^{\prime}(r)\right|\right)^{2}\|u\|^{2} .
\end{aligned}
$$

By the definition of $W(t)$, for all $\omega \in \Lambda$, we have

$$
\begin{aligned}
\|h(v-\epsilon u+W)\|^{2}= & \sum_{i \in \mathbb{Z}^{n}}\left|h_{i}\left(v_{i}-\epsilon u_{i}+a_{i} w_{i}(t, \omega)\right)\right|^{2} \\
= & \sum_{i \in \mathbb{Z}^{n}}\left|h_{i}^{\prime}\left[\theta_{i}\left(v_{i}-\epsilon u_{i}+a_{i} w_{i}(t, \omega)\right)\right]\right|^{2} \\
& \quad \times\left|v_{i}-\epsilon u_{i}+a_{i} w_{i}(t, \omega)\right|^{2} \\
\leq & \beta^{2} \sum_{i \in \mathbb{Z}^{n}}\left|v_{i}-\epsilon u_{i}+a_{i} w_{i}(t, \omega)\right|^{2} \\
\leq & 3 \beta^{2}\left(\|v\|^{2}+\epsilon^{2}\|u\|^{2}+\|W(t, \omega)\|^{2}\right),
\end{aligned}
$$

where $\theta_{i} \in(0,1), i=\left(i_{1}, i_{2}, \ldots, i_{n}\right) \in \mathbb{Z}^{n}$. Hence, we can infer that $f(u), h(v-\epsilon u+W) \in l^{2}$ for all $u=\left(u_{i}\right)_{i \in \mathbb{Z}^{n}}, v=\left(v_{i}\right)_{i \in \mathbb{Z}^{n}} \in$ $l^{2}, \omega \in \Lambda$.

Let $B$ be a bounded set in $F, \varphi_{j}=\left(u_{j}, v_{j}\right)=$ $\left(\left(u_{i}^{(j)}\right),\left(v_{i}^{(j)}\right)\right)_{i \in \mathbb{Z}^{n}} \in B, j=1,2$. Similar to the derivation of (21) and (22), there exists a constant $L(B)$ dependent on the bounded set $B$ such that

$$
\begin{aligned}
\left\|G\left(\varphi_{1}\right)-G\left(\varphi_{2}\right)\right\|_{F}^{2} & =\left\|f\left(u^{(1)}\right)-f\left(u^{(2)}\right)\right\|^{2} \\
& =\sum_{i \in \mathbb{Z}^{n}}\left|f_{i}\left(u_{i}^{(1)}\right)-f_{i}\left(u_{i}^{(2)}\right)\right|^{2} \\
\leq & L(B)\left\|u^{(1)}-u^{(2)}\right\|^{2} \leq L(B)\left\|\varphi_{1}-\varphi_{2}\right\|_{F}^{2}, \\
\left\|E\left(\varphi_{1}\right)-E\left(\varphi_{2}\right)\right\|_{F}^{2} & =\| h\left(v^{(1)}-\epsilon u^{(1)}+W\right) \\
& -h\left(v^{(2)}-\epsilon u^{(2)}+W\right) \|^{2} \\
\leq & 2 \beta^{2}\left(\left\|v^{(1)}-v^{(2)}\right\|^{2}+\epsilon^{2}\left\|u^{(1)}-u^{(2)}\right\|^{2}\right) \\
\leq & 2 \beta^{2}\left(1+\frac{\epsilon^{2}}{\lambda}\right)\left\|\varphi_{1}-\varphi_{2}\right\|_{F}^{2} .
\end{aligned}
$$

The above two inequalities imply that $E$ and $G$ are locally Lipschitz on $F$, and the proof is then complete.

Theorem 9. If $(C 1)-(C 5)$ hold, then, for any initial data $\varphi(0)=$ $\left(u_{0}, u_{10}+\epsilon u_{0}\right)^{T} \in F$, there exists a unique local solution $\varphi(t)=$ $(u(t), v(t))^{T}$ of (19) such that $\varphi \in \mathscr{L}^{2}(\Lambda, C([0, T], F))$, where $T$ is a positive constant. In addition, for all $\omega \in \Lambda$, we have the following estimate:

$$
\begin{aligned}
\sup _{t \in[0, T]}\|\varphi(t)\|_{F}^{2} \leq & M_{2}\|\varphi(0)\|_{F}^{2} \\
& +M_{1} \int_{0}^{T}\left(\|W(t, \omega)\|^{2}+\|W(t, \omega)\|^{2 p+2}\right. \\
& \left.+\|g\|^{2}\right) d t
\end{aligned}
$$

where

$$
\begin{gathered}
M_{1}=\max \left\{\frac{1}{2 \sigma+\alpha}, \frac{c_{2}}{p+1}, \frac{4 n \gamma \lambda+\lambda^{2}+c_{2}^{2}}{\sigma \lambda}\right\}, \\
M_{2}=1+\frac{2}{c_{1} \lambda} \max _{s \in[-\|u(0)\|,\|u(0)\|]}\left|f^{\prime}(s)\right| .
\end{gathered}
$$

Moreover, the solution of (19) depends continuously on the initial data $\varphi(0)$; that is, for each $\omega \in \Lambda$, the mapping $\varphi(0) \in$ $F \mapsto \varphi(\cdot, \omega, \varphi(0)) \in C([0, T], F)$ is continuous.

Proof. Taking the inner product $(\cdot, \cdot)_{F}$ of (19) with $\varphi(t)=$ $(u(t), v(t))^{T}=(u(t), \dot{u}(t)+\epsilon u+W)^{T} \in F$, we have

$$
(\dot{\varphi}, \varphi)_{F}=(C(\varphi), \varphi)_{F}+(E(\varphi), \varphi)_{F}+(G(\varphi), \varphi)_{F} .
$$


Denote $C^{*}(\varphi)=C(\varphi)+(\underset{-h(v-\epsilon u+W)}{0})$ and $E^{*}(\varphi)=$ $\left(\begin{array}{c}W \\ -(\xi A+\epsilon) W\end{array}\right)$.

It is easy to check that

$$
\begin{aligned}
(\dot{\varphi}, \varphi)_{F} & =(C(\varphi), \varphi)_{F}+(E(\varphi), \varphi)_{F}+(G(\varphi), \varphi)_{F} \\
& =\left(C^{*}(\varphi), \varphi\right)_{F}+\left(E^{*}(\varphi), \varphi\right)_{F}+(G(\varphi), \varphi)_{F} .
\end{aligned}
$$

Now, let us estimate the terms of (27). First, we get

$$
\begin{aligned}
\left(C^{*}(\varphi), \varphi\right)_{F} & \left(\left(\begin{array}{c}
v-\epsilon u \\
\epsilon \xi A u-A u-\eta u-\xi A v+\epsilon v-\epsilon^{2} u
\end{array}\right),\left(\begin{array}{l}
u \\
v
\end{array}\right)\right)_{F} \\
& +\left(\left(\begin{array}{c}
0 \\
-h(v-\epsilon u+W)
\end{array}\right),\left(\begin{array}{l}
u \\
v
\end{array}\right)\right)_{F} \\
= & (v-\epsilon u, u)_{\lambda} \\
& +(\epsilon \xi A u-A u-\eta u-\xi A v+\epsilon v, v) \\
& -\epsilon^{2}(u, v)-(h(v-\epsilon u+W), v) .
\end{aligned}
$$

By condition (C3), we have

$$
\begin{aligned}
& \epsilon^{2}(u, v)+(h(v-\epsilon u+W), v) \\
& \quad \geq \alpha\|v\|^{2}-\epsilon(\beta-\epsilon)\|u\|\|v\|+\alpha(W, v) .
\end{aligned}
$$

Noticing that $\gamma=1-\epsilon \overline{\bar{\xi}}$, it follows from (29) that

$$
\begin{aligned}
\left(C^{*}(\varphi), \varphi\right)_{F} \leq & -\sigma\|\varphi\|_{F}-\frac{\alpha}{2}\|v\|^{2}-\alpha(W, v)+(\sigma-\epsilon)\|u\|_{\lambda}^{2} \\
& +\left(\sigma-\epsilon-\frac{\alpha}{2}\right)\|v\|^{2}+\frac{\beta \epsilon}{\sqrt{\lambda}}\|u\|_{\lambda}\|v\| .
\end{aligned}
$$

Letting $\sigma=\alpha \lambda /\left(\sqrt{\beta^{2}+4 \lambda}\left(\beta+\sqrt{\beta^{2}+4 \lambda}\right)\right)$, we can see that $4(\epsilon-\sigma)(\alpha / 2-\epsilon-\sigma)=\left(\beta^{2} \epsilon^{2}\right) / \lambda$, and then

$$
\left(C^{*}(\varphi), \varphi\right)_{F} \leq-\sigma\|\varphi\|_{F}-\frac{\alpha}{2}\|v\|^{2}-\alpha(W, v) .
$$

From Young's inequality, it follows that

$$
\begin{aligned}
\left(E^{*}(\varphi), \varphi\right)_{F} & =\left(\left(\begin{array}{c}
W \\
(-\xi A+\epsilon) W
\end{array}\right),\left(\begin{array}{l}
u \\
v
\end{array}\right)\right)_{F} \\
& =(W, u)_{\lambda}+((-\xi A+\epsilon) W, v) \\
& \leq \frac{1}{2 \sigma}\|W\|_{\lambda}^{2}+\frac{\sigma}{2}\|u\|_{\lambda}^{2}+(-\xi A W, v)+\epsilon(W, v),
\end{aligned}
$$

$$
\begin{aligned}
(G(\varphi), \varphi)_{F} & =\left(\left(\begin{array}{c}
0 \\
f(u)+g
\end{array}\right),\left(\begin{array}{l}
u \\
v
\end{array}\right)\right)_{F} \\
& =(-f(u), v)+(g, v) .
\end{aligned}
$$

By using Young's inequality again, we have

$$
(g, v) \leq \frac{1}{2(2 \sigma+\alpha)}\|g\|^{2}+\frac{2 \sigma+\alpha}{2}\|v\|^{2} .
$$

Based on assumption (C2) and the definition of $\epsilon$, it follows that

$$
\begin{aligned}
-( & f(u), v) \\
= & -(f(u), \dot{u}+\epsilon u-W) \\
= & -(f(u), \dot{u})-\epsilon(f(u), u)+(f(u), W) \\
= & -\sum_{i \in \mathbb{Z}^{n}} f_{i}\left(u_{i}\right) \dot{u}_{i}-\epsilon \sum_{i \in \mathbb{Z}^{n}} f_{i}\left(u_{i}\right) u_{i}+(f(u), W) \\
\leq & -\frac{d}{d t} \sum_{i \in \mathbb{Z}^{n}} V_{i}\left(u_{i}\right)-c_{1} \epsilon \sum_{i \in \mathbb{Z}^{n}} V_{i}\left(u_{i}\right) \\
& +c_{2} \sum_{i \in \mathbb{Z}^{n}}\left|u_{i}\right|^{2 p+1}\left|a_{i} w_{i}(t)\right|+c_{2} \sum_{i \in \mathbb{Z}^{n}}\left|u_{i}\right|\left|a_{i} w_{i}(t)\right| \\
\leq & -\frac{d}{d t} \sum_{i \in \mathbb{Z}^{n}} V_{i}\left(u_{i}\right)-\epsilon c_{2} \sum_{i \in \mathbb{Z}^{n}}\left|u_{i}\right|^{2 p+2} \\
& +c_{2} \frac{2 p+1}{2 p+2} \sum_{i \in \mathbb{Z}^{n}}\left|u_{i}\right|^{2 p+2}+c_{2} \frac{1}{2 p+2}\|W\|^{2 p+2} \\
& +c_{2} \sum_{i \in \mathbb{Z}^{n}}\left|u_{i}\right|\left|a_{i} w_{i}(t)\right| \quad(\text { Hölder's inequality) } \\
& +\frac{d}{d t} \sum_{i \in \mathbb{Z}^{n}} V_{i}\left(u_{i}\right)+c_{2} \frac{1}{2 p+2}\|W\|^{2 p+2} \\
& \sigma u\left\|^{2}+\frac{c_{2}^{2}}{2 \sigma \lambda}\right\| W \|^{2} \quad(\text { Young's inequality). }
\end{aligned}
$$

Combining (31)-(35) with (27), we can calculate that

$$
\begin{aligned}
\frac{d\|\varphi\|_{F}^{2}}{d t}+2 \frac{d}{d t} \sum_{i \in \mathbb{Z}^{n}} V_{i}\left(u_{i}\right) \\
\leq M_{1}\left(\|g\|^{2}+\|W(t)\|^{2}+\|W(t)\|^{2 p+2}\right) \\
\quad+(-\xi A W, v)+\epsilon(W, v)-\alpha(W, v) .
\end{aligned}
$$

Since $\epsilon=\alpha \lambda /(\beta+4 \lambda)$, it is easy to check that $\epsilon<\alpha$. Also by condition $(\mathrm{C} 1)$, one has

$$
(-\xi A W, v)+\epsilon(W, v)-\alpha(W, v)<0 .
$$

Substituting (37) into (36) yields

$$
\begin{aligned}
& \frac{d\|\varphi\|_{F}^{2}}{d t}+2 \frac{d}{d t} \sum_{i \in \mathbb{Z}^{n}} V_{i}\left(u_{i}\right) \\
& \leq M_{1}\left(\|g\|^{2}+\|W(t)\|^{2}+\|W(t)\|^{2 p+2}\right) .
\end{aligned}
$$

From (14) it follows that

$$
\begin{aligned}
\sum_{i \in \mathbb{Z}^{n}} V_{i}\left(u_{i 0}\right) & \leq \frac{1}{c_{1}} \sum_{i \in \mathbb{Z}^{n}} f_{i}\left(u_{i 0}\right) u_{i 0} \\
& \leq \frac{1}{c_{1}} \max _{s \in[-\|u(0)\|,\|u(0)\|]}\left|f_{1}^{\prime}(s)\right|\|u(0)\|^{2},
\end{aligned}
$$


and then a combination of the above inequality and (38) leads to

$$
\begin{aligned}
\|\varphi\|_{F}^{2} \leq & \|\varphi(0)\|_{F}^{2}+\frac{2}{c_{1}} \max _{s \in[-\|u(0)\|,\|u(0)\|]}\left|f^{\prime}(s)\right|\|u(0)\|^{2} \\
& +M_{1} \int_{0}^{t}\left(\|g\|^{2}+\|W(\tau)\|^{2}+\|W(\tau)\|^{2 p+2}\right) d \tau .
\end{aligned}
$$

Hence, one has

$$
\begin{aligned}
\|\varphi\|_{F}^{2} \leq & M_{2}\|\varphi(0)\|_{F}^{2} \\
& +M_{1} \int_{0}^{t}\left(\|g\|^{2}+\|W(\tau)\|^{2}+\|W(\tau)\|^{2 p+2}\right) d \tau,
\end{aligned}
$$

which implies that, for all $\omega \in \Lambda,\|\varphi(t)\|_{F}$ is bounded. So, for any $T \in \mathbb{R}^{+}$, (19) has a global solution on any interval $[0, T]$, and therefore for all $\omega \in \Lambda, T>0$, we have

$$
\begin{aligned}
\sup _{t \in[0, T]}\|\varphi(t)\|_{F}^{2} \\
\leq M_{2}\|\varphi(0)\|_{F}^{2} \\
\quad+M_{1} \int_{0}^{T}\left(\|g\|^{2}+\|W(\tau)\|^{2}+\|W(\tau)\|^{2 p+2}\right) d \tau,
\end{aligned}
$$

which indicates that (22) has a global solution $\varphi \in$ $\mathscr{L}^{2}(\Lambda, C[0, T], F)$.

Next, we show that the solutions of (19) are dependent continuously on initial conditions. Let $\varphi_{i}(0)=\left(u_{0}^{(i)}, u_{10}^{(i)}+\right.$ $\left.\epsilon u_{0}^{(i)}\right) \in F$ and $\left\|\varphi_{i}(0)\right\|_{F}<r_{0}$ and assume $\varphi_{i}(t)=$ $\left(\varphi\left(t, \varphi_{i}(0)\right)\right)=\left(u^{(i)}, \dot{u}^{(i)}+\epsilon u^{(i)}-W(t)\right)(i=1,2)$ are the solutions of (19), where $r_{0}$ is a constant.

Set $R_{0}=M_{2} r_{0}^{2}+M_{1} \int_{0}^{T}\left(\|g\|^{2}+\|W(\tau)\|^{2}+\|W(\tau)\|^{2 p+2}\right) d \tau$. Since $\varphi_{1}(t)$ and $\varphi_{2}(t)$ are the solutions of (19), we have

$$
\begin{aligned}
\frac{d\left(\varphi_{2}(t)-\varphi_{1}(t)\right)}{d t}= & \left(C\left(\varphi_{2}\right)-C\left(\varphi_{1}\right)\right)+\left(E\left(\varphi_{2}\right)-E\left(\varphi_{1}\right)\right) \\
& +\left(G\left(\varphi_{2}\right)-G\left(\varphi_{1}\right)\right) .
\end{aligned}
$$

Taking the inner product of (43) with $\left(\varphi_{2}-\varphi_{1}\right)$ in $F$, we get

$$
\begin{aligned}
\frac{1}{2} \frac{d\left\|\varphi_{2}-\varphi_{1}\right\|_{F}^{2}}{d t}= & \left(C\left(\varphi_{2}\right)-C\left(\varphi_{1}\right), \varphi_{2}-\varphi_{1}\right)_{F} \\
& +\left(E\left(\varphi_{2}\right)-E\left(\varphi_{1}\right), \varphi_{2}-\varphi_{1}\right)_{F} \\
& +\left(G\left(\varphi_{2}\right)-G\left(\varphi_{1}\right), \varphi_{2}-\varphi_{1}\right)_{F}
\end{aligned}
$$

By Lemma 8, we have

$$
\begin{aligned}
\frac{1}{2} \frac{d\left\|\varphi_{2}-\varphi_{1}\right\|_{F}^{2}}{d t} \leq & \left(C\left(\varphi_{2}\right)-C\left(\varphi_{1}\right), \varphi_{2}-\varphi_{1}\right)_{F} \\
& +\beta \sqrt{2\left(1+\frac{\epsilon^{2}}{\lambda}\right)}\left\|\varphi_{2}-\varphi_{1}\right\|_{F}^{2} \\
& +\sqrt{L\left(R_{0}\right)}\left\|\varphi_{2}-\varphi_{1}\right\|_{F}^{2} .
\end{aligned}
$$

It is easy to see the operator $C(\cdot)$ in (19) is a linear operator, and then it follows from assumption (C1) that there exists a positive constant $C_{0}$ such that $\|C\|_{F} \leq C_{0}$, where $C_{0}$ depends only on the constants $\epsilon, \overline{\bar{\xi}},\|A\|_{F}$, and $\overline{\bar{\eta}}$. Hence, (45) implies that

$$
\begin{aligned}
& \frac{d\left\|\varphi_{2}-\varphi_{1}\right\|_{F}^{2}}{d t} \\
& \quad \leq 2\left(C_{0}+\beta \sqrt{2\left(1+\frac{\epsilon^{2}}{\lambda}\right)}+\sqrt{L\left(R_{0}\right)}\right)\left\|\varphi_{2}-\varphi_{1}\right\|_{F}^{2} .
\end{aligned}
$$

Furthermore, by Grownwall inequality, it is clear that

$$
\begin{aligned}
\| \varphi_{2}- & \varphi_{1} \|_{F}^{2} \\
\leq & \left\|\varphi_{2}(0)-\varphi_{1}(0)\right\|_{F}^{2} \\
& \quad \times \exp \left\{2\left(C_{0}+\beta \sqrt{2\left(1+\frac{\epsilon^{2}}{\lambda}\right)}+\sqrt{L\left(R_{0}\right)}\right) t\right\},
\end{aligned}
$$

Hence, we have

$$
\begin{aligned}
& \sup _{t \in[0, T]}\left\|\varphi_{2}-\varphi_{1}\right\|_{F}^{2} \\
& \leq\left\|\varphi_{2}(0)-\varphi_{1}(0)\right\|_{F}^{2} \\
& \quad \times \exp \left\{2\left(C_{0}+\beta \sqrt{2\left(1+\frac{\epsilon^{2}}{\lambda}\right)}+\sqrt{L\left(R_{0}\right)}\right) T\right\},
\end{aligned}
$$

which implies that the solutions of system (19) depend continuously on the initial data. The proof is now complete.

From assumption (C5) and noticing $\theta_{\tau} \omega(t)=\omega(\tau+t)-$ $\omega(\tau)(\tau, t \in \mathbb{R})$, it is easy to see that $\left(\Lambda, \mathscr{F}, \mathbb{P},\left(\theta_{t}\right)_{t \in \mathbb{R}}\right)$ is a metric dynamical system. Also, from the definition of $\left(\theta_{t}\right)_{t \in \mathbb{R}}$, we have

$$
W(t+\tau, \omega)=W\left(t, \theta_{\tau} \omega\right)+W(\tau, \omega), \quad \forall \tau, t \in \mathbb{R}
$$

Now, for any $t \geq 0, \omega \in \Lambda$, we introduce the map from $F$ into $F$ as follows:

$$
s(t, \omega) \varphi(0)=\varphi\left(t, \omega, \varphi_{0}\right),
$$

where $\varphi\left(t, \omega, \varphi_{0}\right)$ is the solution of (19) with initial data $\varphi_{0}$ and $s(\cdot, \omega)$ is continuous for $\omega$ from $[0, \infty] \times F$ to $F$ since the solution of (19) is dependent on initial data continuously.

By (49), (C3), and Theorem 9, it is easy to check that (50) defines a continuous random dynamical system $\{s(t, \omega)\}_{t \geq 0, \omega \in \Lambda} \operatorname{over}\left(\Lambda, \mathscr{F}, \mathbb{P},\left(\theta_{t}\right)_{t \in \mathbb{R}}\right)$. 


\section{Existence of the Absorbing Set}

In this section, we are concerned with the existence of an absorbing set $K(\omega)$ for random dynamical system $s(t, \omega)$ generated by the stochastic system (19). We first introduce an Ornstein-Uhlenbeck process in $l^{2}$ on the metric dynamic $\operatorname{system}\left(\Lambda, \mathscr{F}, \mathbb{P},\left(\theta_{t}\right)_{t \in \mathbb{R}}\right)$ (see $[19,24]$ for details).

Letting $y\left(\theta_{t} \omega\right)=-\epsilon \int_{-\infty}^{0} e^{\epsilon \tau} \theta_{t} \omega(\tau) d \tau(t \in \mathbb{R})$ where $\epsilon=$ $\alpha \lambda /\left(\beta^{2}+4 \lambda\right)$, then $y$ solves the Itô equation

$$
d y+\epsilon y d t=d W(t), \quad \text { for } t \geq 0 .
$$

From the properties of the Ornstein-Uhlenbeck process, we know that there exists a $\theta_{t}$-invariant set $\Lambda_{0} \subset \Lambda$ of full $\mathbb{P}$ measure, and the following properties hold:

(Y1) the mapping $s \rightarrow y\left(\theta_{s} \omega\right)$ is continuous for each $\omega \epsilon$ $\Lambda_{0}$;

(Y2) the random variable $\|y(\omega)\|$ is tempered;

(Y3) there exists a tempered function $b(\omega)>0$ such that

$$
\left\|y\left(\theta_{t} \omega\right)\right\|^{2 p+2}+\left\|y\left(\theta_{t} \omega\right)\right\|^{2} \leq b\left(\theta_{t} \omega\right) \leq b(\omega) e^{(\epsilon / 2)|t|} .
$$

Theorem 10. There exist a $\theta_{t}$-invariant set $\Lambda_{0} \subset \Lambda$ of full $\mathbb{P}$ measure and an absorbing set $K(\omega), \omega \in \Lambda_{0}$ for $s(t, \omega) \psi_{0}$. That is, for all $B \in \mathscr{D}$ and all $\omega \in \Lambda_{0}$, there exists $T_{B}(\omega)>0$ such that

$$
s\left(t, \theta_{-t} \omega\right) B\left(\theta_{-t} \omega\right) \subset K(\omega), \quad \forall t>T_{B}(\omega) .
$$

Moreover, $K \in \mathscr{D}$; that is, for all $\omega \in \Lambda_{0}$, there exists $T_{K}(\omega)$ such that

$$
s\left(t, \theta_{-t} \omega\right) K\left(\theta_{-t} \omega\right) \subset K(\omega), \quad \forall t>T_{K}(\omega) .
$$

Proof. Letting $\psi(t)=\psi(t)=\left(u(t), v^{*}(t)\right)^{T}=(u(t), \dot{u}(t)+$ $\left.\epsilon u(t)-y\left(\theta_{t} \omega\right)\right)^{T}=\varphi(t)+\left(0, W(t, \omega)-y\left(\theta_{t} \omega\right)\right)^{T}$, where $\varphi(t)$ is a solution of (19), then, for any $\omega \in \Lambda, y(\omega)$ has properties (Y1), (Y2), and (Y3). By the Itô equation (51), it can be inferred that $\psi(t)$ satisfies

$$
\begin{aligned}
\frac{d \psi(t)}{d t}= & C(\psi)+G(\psi) \\
& +\left(\begin{array}{c}
y\left(\theta_{t} \omega\right) \\
-h\left(v^{*}-\epsilon u+y\right)+(-\xi A+2 \epsilon) y\left(\theta_{t} \omega\right)
\end{array}\right) .
\end{aligned}
$$

Taking the inner product of (55) with $\psi$ in $F$, we obtain

$$
\begin{aligned}
\frac{1}{2} \frac{d\|\psi(t)\|_{F}^{2}}{d t} & \\
= & (C(\psi), \psi)_{F}+(G(\psi), \psi)_{F} \\
& +\left(\left(\begin{array}{c}
y\left(\theta_{t} \omega\right) \\
-h\left(v^{*}-\epsilon u+y\right)+(-\xi A+2 \epsilon) y\left(\theta_{t} \omega\right)
\end{array}\right),\left(\begin{array}{c}
u \\
v^{*}
\end{array}\right)\right)_{F} \\
= & \left(C^{*}(\varphi), \varphi\right)_{F}+\left(E^{*}(\varphi), \varphi\right)_{F}+\left(G^{*}(\varphi), \varphi\right)_{F} .
\end{aligned}
$$

Similar to the derivation of (31) and (32), we have

$$
\begin{aligned}
\left(C^{*}(\psi), \psi\right)_{F}= & (C(\psi), \psi)_{F} \\
& +\left(\left(\begin{array}{c}
0 \\
-h\left(v^{*}-\epsilon u+y\right)
\end{array}\right),\left(\begin{array}{c}
u \\
v^{*}
\end{array}\right)\right)_{F} \\
\leq & -\sigma\|\psi\|_{F}^{2}-\frac{\alpha}{2}\left\|v^{*}\right\|^{2}-\alpha\left(y\left(\theta_{t} \omega\right), v^{*}\right) ; \\
\left(E^{*}(\psi), \psi\right)_{F} \leq & \frac{1}{\sigma}\left\|y\left(\theta_{t} \omega\right)\right\|_{\lambda}^{2}+\frac{\sigma}{4}\|u\|_{\lambda}^{2} \\
& +\left(-\xi A y\left(\theta_{t} \omega\right), v^{*}\right)+\epsilon\left(y\left(\theta_{t} \omega\right), v^{*}\right),
\end{aligned}
$$

where $\sigma, E^{*}(\cdot)$, and $C^{*}(\cdot)$ are defined in (29) and (26), respectively.

Notice that

$$
\begin{aligned}
(G(\psi), \psi)_{F} & =\left(\left(\begin{array}{c}
0 \\
f(u)+g
\end{array}\right),\left(\begin{array}{c}
u \\
v^{*}
\end{array}\right)\right)_{F} \\
& =\left(-f(u), v^{*}\right)+\left(g, v^{*}\right) \\
& =\left(-f(u), \dot{u}+\epsilon u-y\left(\theta_{t} \omega\right)\right)+\left(g, v^{*}\right) .
\end{aligned}
$$

Similar to the derivation of (35), by condition (C2), (17), and $(4 p+2) /(4 p+3) \leq \epsilon \leq 1$, one has

$$
\begin{aligned}
\left(-f(u), \dot{u}+\epsilon u-y\left(\theta_{t} \omega\right)\right) & (-f(u), \dot{u})-\epsilon(f(u), u)+\left(f(u), y\left(\theta_{t} \omega\right)\right) \\
\leq & -\frac{d}{d t} \sum_{i \in \mathbb{Z}^{n}} V_{i}\left(u_{i}\right)-c_{1} \epsilon \sum_{i \in \mathbb{Z}^{n}} V_{i}\left(u_{i}\right) \\
& +c_{2} \sum_{i \in \mathbb{Z}^{n}}\left|u_{i}\right|^{2 p+1}\left|a_{i} y_{i}\left(\theta_{t} \omega\right)\right| \\
& +c_{2} \sum_{i \in \mathbb{Z}^{n}}\left|u_{i}\right|\left|a_{i} y_{i}\left(\theta_{t} \omega\right)\right| \\
\leq & -\frac{d}{d t} \sum_{i \in \mathbb{Z}^{n}} V_{i}\left(u_{i}\right)-\frac{c_{1} \epsilon}{4 p+4} \sum_{i \in \mathbb{Z}^{n}} V_{i}\left(u_{i}\right) \\
& -\frac{c_{2} \epsilon(4 p+3)}{4 p+4} \sum_{i \in \mathbb{Z}^{n}}\left|u_{i}\right|^{2 p+2} \\
& +\frac{c_{2}}{2 p+2}\|y\|^{2 p+2}+\frac{\sigma \lambda}{4}\|u\|^{2}+\frac{c_{2}^{2}}{\sigma \lambda}\|y\|^{2}, \\
& +\frac{c_{2}(2 p+1)}{2 p+2} \sum_{i \in \mathbb{Z}^{n}}\left|u_{i}\right|^{2 p+2} \\
& +\frac{c_{2}}{2 p+2}\|y\|^{2 p+2}+\frac{\sigma \lambda}{4 t}\|u\|^{2}+\frac{c_{2}^{2}}{\sigma \lambda}\|y\|^{2} \\
& +V_{i}\left(u_{i}\right)-\frac{c_{1} \epsilon}{4 p+4} \sum_{i \in \mathbb{Z}^{n}} V_{i}\left(u_{i}\right) \\
& \\
& \\
&
\end{aligned}
$$

and, by Young's inequality, we also have

$$
\left(g, v^{*}\right) \leq \frac{1}{2(\sigma+\alpha)}\|g\|^{2}+\frac{\sigma+\alpha}{2}\left\|v^{*}\right\|^{2} .
$$


Substituting (57)-(61) into (56), we obtain

$$
\begin{aligned}
\frac{d}{d t}\left(\|\psi\|_{F}^{2}+2 \sum_{i \in \mathbb{Z}^{n}} V_{i}\left(u_{i}\right)\right) \\
\leq-\sigma\|\psi\|_{F}^{2}-2 c_{1} \epsilon \sum_{i \in \mathbb{Z}^{n}} V_{i}\left(u_{i}\right)+\frac{1}{\sigma+\alpha}\|g\|^{2} \\
\quad+M_{3}\left(\left\|y\left(\theta_{t} \omega\right)\right\|^{2}+\left\|y\left(\theta_{t} \omega\right)\right\|^{2 p+2}\right) \\
\quad+\left(-\xi A y\left(\theta_{t} \omega\right), v^{*}\right)+2 \epsilon\left(y\left(\theta_{t} \omega\right), v^{*}\right)-\alpha\left(y\left(\theta_{t} \omega\right), v^{*}\right) .
\end{aligned}
$$
then

By the definition of constant $\epsilon$, we find that $2 \epsilon<\alpha$, and

$$
\left(-\xi A y\left(\theta_{t} \omega\right), v^{*}\right)+2 \epsilon\left(y\left(\theta_{t} \omega\right), v^{*}\right)-\alpha\left(y\left(\theta_{t} \omega\right), v^{*}\right)<0 .
$$

Thus, we arrive at

$$
\begin{aligned}
\frac{d}{d t}( & \left.\|\psi\|_{F}^{2}+2 \sum_{i \in \mathbb{Z}^{n}} V_{i}\left(u_{i}\right)\right) \\
\leq & -\sigma\|\psi\|_{F}^{2}-2 c_{1} \epsilon \sum_{i \in \mathbb{Z}^{n}} V_{i}\left(u_{i}\right)+\frac{1}{\sigma+\alpha}\|g\|^{2} \\
& +M_{3}\left(\left\|y\left(\theta_{t} \omega\right)\right\|^{2}+\left\|y\left(\theta_{t} \omega\right)\right\|^{2 p+2}\right) .
\end{aligned}
$$

Furthermore, from Grownwall inequality, it follows that

$$
\begin{aligned}
\|\psi\|_{F}^{2} \leq & M_{2}\left\|\psi_{0}\right\|_{F}^{2} e^{-c_{3} t}+\frac{1}{c_{3}(\sigma+\alpha)}\|g\|^{2} \\
& +M_{3} \int_{0}^{t} e^{c_{3}(s-t)}\left(\left\|y\left(\theta_{s} \omega\right)\right\|^{2}+\left\|y\left(\theta_{s} \omega\right)\right\|^{2 p+2}\right) d s,
\end{aligned}
$$

where

$$
\begin{gathered}
M_{3}=\max \left\{\frac{c_{2}}{p+1}, \frac{4 n \gamma \lambda+\lambda^{2}+c_{2}^{2}}{\sigma \lambda}\right\}, \\
c_{3}=\min \left\{\sigma, \frac{c_{1} \epsilon}{4 p+4}\right\} .
\end{gathered}
$$

Therefore, it follows from (65) and property (Y3) that

$$
\begin{aligned}
\| \psi( & \left.t, \theta_{-t} \omega, \psi_{0}\left(\theta_{-t} \omega\right)\right) \|_{F}^{2} \\
\leq & M_{2}\left\|\psi_{0}\left(\theta_{-t} \omega\right)\right\|_{F}^{2} e^{-c_{3} t}+\frac{1}{c_{3}(\sigma+\alpha)}\|g\|^{2} \\
& +M_{3} \int_{0}^{t} e^{c_{3}(s-t)}\left(\left\|y\left(\theta_{s-t} \omega\right)\right\|^{2}+\left\|y\left(\theta_{s-t} \omega\right)\right\|^{2 p+2}\right) d s \\
\leq & M_{2}\left\|\psi_{0}\left(\theta_{-t} \omega\right)\right\|_{F}^{2} e^{-c_{3} t}+\frac{1}{c_{3}(\sigma+\alpha)}\|g\|^{2} \\
& +M_{3} \int_{-t}^{0} e^{c_{3} \tau}\left(\left\|y\left(\theta_{\tau} \omega\right)\right\|^{2}+\left\|y\left(\theta_{\tau} \omega\right)\right\|^{2 p+2}\right) d \tau
\end{aligned}
$$

$$
\begin{aligned}
\leq & M_{2}\left\|\psi_{0}\left(\theta_{-t} \omega\right)\right\|_{F}^{2} e^{-c_{3} t}+\frac{1}{c_{3}(\sigma+\alpha)}\|g\|^{2} \\
& +M_{3} \int_{-t}^{0} e^{c_{3} \tau} b(\omega) e^{(\epsilon / 2)|\tau|} d \tau \\
\leq & M_{2}\left\|\psi_{0}\left(\theta_{-t} \omega\right)\right\|_{F}^{2} e^{-c_{3} t}+\frac{1}{c_{3}(\sigma+\alpha)}\|g\|^{2} \\
& +\frac{2 M_{3}}{2 c_{3}+\epsilon} b(\omega) \\
\leq & M_{2}\left\|\psi_{0}\left(\theta_{-t} \omega\right)\right\|_{F}^{2} e^{-c_{3} t}+\widetilde{B}(\omega),
\end{aligned}
$$

where $\widetilde{B}(\omega)=\left(1 / c_{3}(\sigma+\alpha)\right)\|g\|^{2}+\left(2 M_{3} /\left(2 c_{3}+\epsilon\right)\right) b(\omega)$ and $\widetilde{B}(\omega)$ is tempered thanks to the tempered function $b(\omega)$. Then, $\widetilde{K}(\omega)=\left\{\psi \in F,\|\psi\|_{F} \leq \widetilde{B}(\omega)\right\}$ is an absorbing set for $\psi(t, \omega, \psi(0))$; that is, for all $B \in \mathscr{D}$ and all $\omega \in \Lambda_{0}$, there exists $T_{B}(\omega)>0$ such that $\psi\left(t, \theta_{-t}, B\left(\theta_{-t} \omega\right)\right) \subset \widetilde{K}(\omega)$ for all $t \geq T_{B}(\omega)$.

Let $K(\omega)=\left\{\varphi \in F,\|\varphi\|_{F} \leq \widetilde{B}(\omega)+\|y(\omega)\|\right\}$; then $K(\omega)$ is an absorbing set for $\varphi\left(t, \omega, \varphi_{0}\right)=\psi\left(t, \omega, \varphi_{0}-(0, y(\omega))^{T}-\right.$ $\left.\left(0, y\left(\theta_{t} \omega\right)-W(t, \omega)\right)^{T}\right)$. Moreover, $K \in \mathscr{D}$. The proof is now complete.

\section{Existence of a Global Random Attractor}

In this section, we will show the existence of global random attractor related to the random lattice dynamical system $s(t, \omega)$ generated by system (19). In order to apply the result of Theorem 5, we need to prove the following lemmas.

Lemma 11. Let (C1)-(C5) hold and $\varphi(0)=\left(u_{0}, u_{10}+\epsilon u_{0}\right) \epsilon$ $K(\omega)$. Then, for any $\mu>0$, there exist $T(\mu, \omega)>0$ and $N(\mu, \omega)>0$ such that the solution of (19) satisfies

$$
\sum_{\|i\|_{m} \geq N(\mu, \omega)}\left\|\varphi_{i}\left(t, \theta_{-t} \omega, \varphi_{0}\left(\theta_{-t} \omega\right)\right)\right\|_{F}^{2} \leq \mu, \quad \forall t \geq T(\mu, \omega),
$$

where $\|i\|_{m}=\max _{1 \leq j \leq n}\left|i_{j}\right|$, for any $i=\left(i_{1}, i_{2}, \ldots, i_{n}\right) \in \mathbb{Z}^{n}$.

Proof. Assume $\vartheta \in C^{1}\left(R^{+}, R\right)$ is a smooth function satisfying

$$
\begin{gathered}
\vartheta(t)=0, \quad 0 \leq t \leq 1 ; \\
0 \leq \vartheta(t) \leq 1, \quad 1 \leq t \leq 2 ; \\
\vartheta(t)=1, \quad t \geq 2,
\end{gathered}
$$

and there exists a constant $M_{0}$ such that $\left|\vartheta^{\prime}(t)\right| \leq M_{0}$ for $t \epsilon$ $R^{+}$.

Let $\psi(t)=\left(u(t), v^{*}(t)\right)^{T}=\left(u_{i}(t), v_{i}^{*}(t)\right)_{i \in \mathbb{Z}^{n}}^{T}$ be a solution of (55), where $v^{*}(t)=\dot{u}(t)+\epsilon u(t)-y\left(\theta_{t} \omega\right), \omega \in \Lambda_{0}$.

Suppose $M$ is a suitable large constant to be defined later. Set $\widetilde{\psi}=(\widetilde{w}, \widetilde{z})=\left(\widetilde{w}_{i}, \widetilde{z}_{i}\right)_{i \in \mathbb{Z}^{n}}$, where

$$
\widetilde{w}_{i}=\vartheta\left(\frac{\|i\|_{m}}{M}\right) u_{i}, \quad \widetilde{z}_{i}=\vartheta\left(\frac{\|i\|_{m}}{M}\right) v_{i}^{*}
$$

for all $i \in \mathbb{Z}^{n}$. 
Taking the inner product $(\cdot, \cdot)_{F}$ of (55) with $\widetilde{\psi}$, we have

$$
\begin{aligned}
\left(\frac{d \psi(t)}{d t}, \widetilde{\psi}(t)\right)_{F}= & \left(C^{*}(\psi), \widetilde{\psi}\right)_{F}+(G(\psi), \widetilde{\psi})_{F} \\
& +\left(\left(\begin{array}{c}
y\left(\theta_{t} \omega\right) \\
(-\xi A+2 \epsilon) y\left(\theta_{t} \omega\right)
\end{array}\right),\left(\begin{array}{c}
\widetilde{w} \\
\widetilde{z}
\end{array}\right)\right)_{F}
\end{aligned}
$$

It is easy to see

$$
\begin{aligned}
&\left(\frac{d \psi(t)}{d t}, \widetilde{\psi}(t)\right)_{F}=\frac{1}{2} \frac{d}{d t} \sum_{i \in \mathbb{Z}^{n}} \vartheta\left(\frac{\|i\|_{m}}{M}\right)\left\|\psi_{i}(t)\right\|_{F}^{2},(7 \\
&\left(C^{*}(\psi), \widetilde{\psi}\right)_{F} \\
&=(C(\psi), \widetilde{\psi})_{F}+\left(\left(\begin{array}{c}
0 \\
-h\left(v^{*}-\epsilon u+y\right)
\end{array}\right),\left(\frac{\widetilde{w}}{\widetilde{z}}\right)\right)_{F} \\
&=\left(v^{*}-\epsilon u, \widetilde{w}\right)_{\lambda} \\
& \quad+\left(\epsilon \xi A u-A u-\eta u-\xi A v^{*}+\epsilon v^{*}-\epsilon^{2} u, \widetilde{z}\right) \\
& \quad+\left(-h\left(v^{*}-\epsilon u+y\right), \widetilde{z}\right) \\
& \leq \gamma \sum_{j=1}^{n}\left(D_{j} v^{*}, D_{j} \widetilde{w}\right)+\lambda\left(v^{*}, \widetilde{w}\right)-\epsilon \gamma \sum_{j=1}^{n}\left(D_{j} u, D_{j} \widetilde{w}\right) \\
&-\epsilon \lambda(u, \widetilde{w})+\epsilon \overline{\bar{\xi}} \sum_{j=1}^{n}\left(D_{j} u, D_{j} \widetilde{z}\right) \\
&-\sum_{j=1}^{n}\left(D_{j} u, D_{j} \widetilde{z}\right)-\lambda(u, \widetilde{z})-\bar{\xi} \sum_{j=1}^{n}\left(D_{j} v^{*}, D_{j} \widetilde{z}\right) \\
& \quad+\lambda\left(v^{*}, \widetilde{w}\right)-\epsilon \gamma \sum_{j=1}^{n}\left(D_{j} u, D_{j} \widetilde{w}\right) \\
&-\epsilon^{2}(u, \widetilde{z})+\left(-h\left(v^{*} v^{*}, D_{j} \widetilde{w}\right)+(\epsilon u+\overline{\bar{\xi}}-1) \sum_{j=1}^{n}\left(D_{j} u, D_{j} \widetilde{z}\right)\right. \\
&
\end{aligned}
$$

$$
\begin{aligned}
& \gamma \sum_{j=1}^{n}\left(D_{j} v^{*}, D_{j} \widetilde{w}\right)+(\epsilon \overline{\bar{\xi}}-1) \sum_{j=1}^{n}\left(D_{j} u, D_{j} \widetilde{z}\right) \\
& =\gamma \sum_{j=1}^{n} \sum_{i \in \mathbb{Z}^{n}}\left\{\left[\vartheta\left(\frac{\left\|i_{j}\right\|_{m}}{M}\right) u_{i_{j}}\left(v_{i_{j}}^{*}-v_{i}^{*}\right)\right.\right. \\
& \left.-\vartheta\left(\frac{\|i\|_{m}}{M}\right) u_{i}\left(v_{i_{j}}^{*}-v_{i}^{*}\right)\right] \\
& -\left[\vartheta\left(\frac{\left\|i_{j}\right\|_{m}}{M}\right) v_{i_{j}}^{*}\left(u_{i_{j}}-u_{i}\right)\right. \\
& \left.\left.-\vartheta\left(\frac{\|i\|_{m}}{M}\right) v_{i_{j}}^{*}\left(u_{i_{j}}-u_{i}\right)\right]\right\} \\
& \leq \gamma \sum_{j=1}^{n} \sum_{i \in \mathbb{Z}^{n}}\left[\vartheta\left(\frac{\left\|i_{j}\right\|_{m}}{M}\right)-\vartheta\left(\frac{\|i\|_{m}}{M}\right)\right]\left(u_{i_{j}} v_{i}-u_{i} v_{i_{j}}\right) \\
& \leq \frac{\gamma M_{0}}{M} \gamma \sum_{j=1 i \in \mathbb{Z}^{n}}^{n} \sum_{i_{i}}\left(v_{i}-u_{i} v_{i_{j}}\right) \\
& \leq \frac{2 n \gamma M_{0}}{M}\|u\|\|v\|, \quad \forall t \in[0, T], T>0, \omega \in \Lambda_{0},
\end{aligned}
$$$$
\sum_{j=1}^{n}\left(D_{j} u, D_{j} \widetilde{w}\right)
$$$$
=\sum_{j=1}^{n} \sum_{i \in \mathbb{Z}^{n}}\left\{\left[\vartheta\left(\frac{\left\|i_{j}\right\|_{m}}{M}\right)-\vartheta\left(\frac{\|i\|_{m}}{M}\right)\right] u_{i_{j}}\left(u_{i}-u_{i_{j}}\right)\right.
$$$$
\left.+\vartheta\left(\frac{\|i\|_{m}}{M}\right)\left(u_{i_{j}}-u_{i}\right)^{2}\right\}
$$$$
\geq-\frac{2 n M_{0}}{M}\|u\|^{2}, \quad T>0, \omega \in \Lambda_{0} .
$$

Also, similar to the derivation of (75), we get

$$
\begin{aligned}
& \sum_{j=1}^{n}\left(D_{j} u, D_{j} \tilde{z}\right) \\
& \quad=\sum_{j=1}^{n} \sum_{i \in \mathbb{Z}^{n}}\left\{\left[\vartheta\left(\frac{\left\|i_{j}\right\|_{m}}{M}\right)-\vartheta\left(\frac{\|i\|_{m}}{M}\right)\right] v_{i_{j}}^{*}\left(v_{i}^{*}-v_{i_{j}}^{*}\right)\right.
\end{aligned}
$$

where the last inequality follows from assumption (C1), (9), and the definition of operator $A$ in (8).
For simplicity, we denote $i_{j}=\left(i_{1}, \ldots, i_{j}+1, \ldots, i_{n}\right)$ and $i=\left(i_{1}, \ldots, i_{j}, \ldots, i_{n}\right)$. Since $\gamma=1-\epsilon \overline{\bar{\xi}}$, by the definition of $\vartheta$, we have 


$$
\begin{array}{r}
+\vartheta\left(\frac{\|i\|_{m}}{M}\right)\left(v_{i_{j}}^{*}-v_{i}^{*}\right)^{2} \\
\geq-\frac{2 n M_{0}}{M}\left\|v^{*}\right\|^{2}, \quad T>0, \omega \in \Lambda_{0},
\end{array}
$$

where $\left\|i_{j}\right\|_{m}=\max \left\{i_{j}+1,\|i\|_{m}\right\}$.

It is not difficult to see

$$
\begin{gathered}
\left(v^{*}, \widetilde{w}\right)=\sum_{i \in \mathbb{Z}^{n}} \vartheta\left(\frac{\|i\|_{m}}{M}\right)\left|u_{i}\right|\left|v_{i}^{*}\right|, \\
(u, \widetilde{w})=\sum_{i \in \mathbb{Z}^{n}} \vartheta\left(\frac{\|i\|_{m}}{M}\right)\left|u_{i}\right|^{2}, \\
(u, \widetilde{z})=\sum_{i \in \mathbb{Z}^{n}} \vartheta\left(\frac{\|i\|_{m}}{M}\right)\left|u_{i}\right|\left|v_{i}^{*}\right|, \\
\left(v^{*}, \widetilde{z}\right)=\sum_{i \in \mathbb{Z}^{n}} \vartheta\left(\frac{\|i\|_{m}}{M}\right)\left|v_{i}^{*}\right|^{2} .
\end{gathered}
$$

Substituting (74)-(80) into (73) yields

$$
\begin{aligned}
\left(C^{*}(\psi), \psi\right)_{F} & \\
\leq & \frac{2 n \gamma M_{0}}{M}\|u\|\|v\|+\frac{2 n M_{0} \epsilon \gamma}{M}\|u\|^{2}+\frac{2 n M_{0} \bar{\xi}}{M}\left\|v^{*}\right\|^{2} \\
& \quad-\sum_{i \in \mathbb{Z}^{n}} \vartheta\left(\frac{\|i\|_{m}}{M}\right)\left[\sigma\left\|\psi_{i}\right\|_{F}^{2}+\frac{\alpha}{2}\left|v_{i}^{*}\right|^{2}\right]-\alpha(y, \widetilde{z}) .
\end{aligned}
$$

Also, it is obvious that

$$
\begin{aligned}
\left(E^{*}(\psi), \widetilde{\psi}\right)_{F}= & \left(\left(\begin{array}{c}
y\left(\theta_{t} \omega\right) \\
(-\xi A+2 \epsilon) y\left(\theta_{t} \omega\right)
\end{array}\right),\left(\begin{array}{c}
\widetilde{w} \\
\widetilde{z}
\end{array}\right)\right)_{F} \\
= & \sum_{i \in \mathbb{Z}^{n}}\left(\vartheta\left(\frac{\|i\|_{m}}{M}\right) u_{i}, y_{i}\right)_{\lambda} \\
& +\left((-\xi A+2 \epsilon) y\left(\theta_{t} \omega\right), \widetilde{z}\right) .
\end{aligned}
$$

$$
\begin{aligned}
(G(\psi), \widetilde{\psi})_{F}= & \left(\left(\begin{array}{c}
0 \\
f(u)+g
\end{array}\right),\left(\begin{array}{c}
\widetilde{w} \\
\widetilde{z}
\end{array}\right)\right)_{F} \\
= & (f(u), \widetilde{z})+(g, \widetilde{z}) \\
= & \sum_{i \in \mathbb{Z}^{n}}\left(f_{i}\left(u_{i}\right), \vartheta\left(\frac{\|i\|_{m}}{M}\right) \dot{u}_{i}\right) \\
& +\sum_{i \in \mathbb{Z}^{n}}\left(f_{i}\left(u_{i}\right), \vartheta\left(\frac{\|i\|_{m}}{M}\right) u_{i}\right) \\
& -\sum_{i \in \mathbb{Z}^{n}}\left(f_{i}\left(u_{i}\right), \vartheta\left(\frac{\|i\|_{m}}{M}\right) y_{i}\right) \\
& +\sum_{i \in \mathbb{Z}^{n}}\left(g_{i}, \vartheta\left(\frac{\|i\|_{m}}{M}\right) v_{i}^{*}\right) .
\end{aligned}
$$

By assumption (C2), we have

$$
\sum_{i \in \mathbb{Z}^{n}}\left(f_{i}\left(u_{i}\right), \vartheta\left(\frac{\|i\|_{m}}{M}\right) \dot{u}_{i}\right)=\frac{d}{d t} \sum_{i \in \mathbb{Z}^{n}} \vartheta\left(\frac{\|i\|_{m}}{M}\right) V_{i}\left(u_{i}\right),
$$

$$
\begin{gathered}
\sum_{i \in \mathbb{Z}^{n}}\left(f_{i}\left(u_{i}\right), \vartheta\left(\frac{\|i\|_{m}}{M}\right) u_{i}\right)=\sum_{i \in \mathbb{Z}^{n}} \vartheta\left(\frac{\|i\|_{m}}{M}\right) V_{i}\left(u_{i}\right), \\
\sum_{i \in \mathbb{Z}^{n}}\left(f_{i}\left(u_{i}\right), \vartheta\left(\frac{\|i\|_{m}}{M}\right) y_{i}\right) \\
\leq \sum_{i \in \mathbb{Z}^{n}} \vartheta\left(\frac{\|i\|_{m}}{M}\right)\left[c_{2}\left(\left|u_{i}\right|^{2 p+2}\left|y_{i}\right|+\left|u_{i}\right|\left|y_{i}\right|\right)\right] .
\end{gathered}
$$

Furthermore, by Young's inequality, we know

$$
\begin{gathered}
\sum_{i \in \mathbb{Z}^{n}}\left(g_{i}, \vartheta\left(\frac{\|i\|_{m}}{M}\right) v_{i}^{*}\right) \\
\leq \frac{\sigma+\alpha}{2} \sum_{\|i\|_{m} \geq M} \vartheta\left(\frac{\|i\|_{m}}{M}\right)\left|v_{i}^{*}\right|^{2}+\frac{1}{2(\sigma+\alpha)} \sum_{\|i\|_{m} \geq M} g_{i}^{2}, \\
\|u\|_{\lambda}\|v\| \leq \frac{1}{2 \sqrt{\lambda}}\|u\|_{\lambda}^{2}+\frac{\sqrt{\lambda}}{2}\|v\|^{2} .
\end{gathered}
$$

Substituting (72), (81)-(88) into (71) leads to

$$
\begin{aligned}
\frac{d}{d t}\left[\sum_{i \in \mathbb{Z}^{n}} \vartheta\left(\frac{\|i\|_{m}}{M}\right)\left(\left\|\psi_{i}\right\|_{F}^{2}+2 V_{i}\left(u_{i}\right)\right)\right] \\
\leq-\sigma \sum_{i \in \mathbb{Z}^{n}} \vartheta\left(\frac{\|i\|_{m}}{M}\right)\left\|\psi_{i}\right\|_{F}^{2} \\
\quad-2 c_{1} \epsilon \sum_{i \in \mathbb{Z}^{n}} \vartheta\left(\frac{\|i\|_{m}}{M}\right)\left(V_{i}\left(u_{i}\right)\right) \\
+\frac{4 \gamma M_{0} n}{M \sqrt{\lambda}}\|u\|_{\lambda}\|v\|+\frac{4 M_{0} n \epsilon \gamma}{M \lambda}\|u\|_{\lambda}+\frac{2 M_{0} n \bar{\xi}}{M}\left\|v^{*}\right\|^{2} \\
+M_{4}\left(\sum_{\|i\|_{m} \geq M}\left|y_{i}\right|^{2}+\sum_{\|i\|_{m} \geq M}\left|y_{i}\right|^{2 p+2}\right) \\
+\frac{1}{\sigma+\alpha} \sum_{\|i\|_{m} \geq M}\left|g_{i}\right|^{2},
\end{aligned}
$$

where $M_{4}$ only depends on $\alpha, \beta, n, p, \lambda$, and $c_{2}$.

Now, setting $M_{5}=\max \left\{2 r M_{0} n(1+\epsilon) / M \lambda, M_{0} n(2 \gamma+\right.$ $\bar{\xi}) / M\}$, we estimate

$$
\begin{aligned}
\frac{d}{d t} & {\left[\sum_{i \in \mathbb{Z}^{n}} \vartheta\left(\frac{\|i\|_{m}}{M}\right)\left(\left\|\psi_{i}\right\|_{F}^{2}+2 V_{i}\left(u_{i}\right)\right)\right] } \\
& \leq-\sigma \sum_{i \in \mathbb{Z}^{n}} \vartheta\left(\frac{\|i\|_{m}}{M}\right)\left\|\psi_{i}\right\|_{F}^{2}-2 c_{1} \epsilon \sum_{i \in \mathbb{Z}^{n}} \vartheta\left(\frac{\|i\|_{m}}{M}\right) V_{i}\left(u_{i}\right)
\end{aligned}
$$




$$
\begin{aligned}
& +M_{5}\left\|\psi_{i}\right\|_{F}^{2}+\frac{1}{\sigma+\alpha} \sum_{\|i\|_{m} \geq M}\left|g_{i}\right|^{2} \\
& +M_{4}\left(\sum_{\|i\|_{m} \geq M}\left|y_{i}\right|^{2}+\sum_{\|i\|_{m} \geq M}\left|y_{i}\right|^{2 p+2}\right) .
\end{aligned}
$$

By Grownwall inequality, for all $t \geq T_{K}=T_{K}(\omega)$, we can deduce that

$$
\begin{aligned}
\sum_{i \in \mathbb{Z}^{n}} \vartheta & \left(\frac{\|i\|_{m}}{M}\right)\left\|\psi_{i}\left(t, \omega, \psi_{0}(\omega)\right)\right\|_{F}^{2} \\
\leq & M_{2} e^{-c_{\mathcal{B}}\left(t-T_{K}\right)} \sum_{i \in \mathbb{Z}^{n}} \vartheta\left(\frac{\|i\|_{m}}{M}\right)\left\|\psi_{i}\left(T_{K}, \omega, \psi_{0}(\omega)\right)\right\|_{F}^{2} \\
& +M_{5} \int_{T_{K}}^{t} e^{c_{3}(\tau-t)}\left\|\psi\left(\tau, \omega, \psi_{0}(\omega)\right)\right\|_{F}^{2} d \tau \\
& +\frac{1}{c_{\mathcal{B}}(\sigma+\alpha)} \sum_{\|i\|_{m} \geq M}\left|g_{i}\right|^{2}+M_{4} \int_{T_{K}}^{t} e^{c_{3}(\tau-t)} \\
& \times \sum_{\|i\|_{m} \geq M}\left(\left|y_{i}\left(\theta_{\tau} \omega\right)\right|^{2 p+2}+\left|y_{i}\left(\theta_{\tau} \omega\right)\right|^{2}\right) d \tau
\end{aligned}
$$

where $c_{3}, M_{2}, M_{4}$, and $M_{5}$ are defined above.

Next, let us estimate each term on the right hand side of (91). From (65) in Theorem 10 and property (Y3), we find that

$$
\begin{gathered}
e^{-c_{3}\left(t-T_{K}\right)} \sum_{i \in \mathbb{Z}^{n}} \vartheta\left(\frac{\|i\|_{m}}{M}\right)\left\|\psi_{i}\left(T_{k}, \theta_{-t} \omega, \psi_{0}\left(\theta_{-t} \omega\right)\right)\right\|_{F}^{2} \\
\leq e^{-c_{3}\left(t-T_{K}\right)}\left[M_{2}\left\|\psi_{0}\left(\theta_{-t} \omega\right)\right\|_{F}^{2} e^{-c_{3} T_{K}}+\frac{1}{c_{3}(\sigma+\alpha)}\|g\|^{2}\right. \\
+M_{3} \int_{0}^{T_{K}} e^{c_{3}\left(s-T_{K}\right)}\left(\left\|y\left(\theta_{s-t} \omega\right)\right\|^{2 p+2}\right. \\
\left.\left.+\left\|y\left(\theta_{s-t} \omega\right)\right\|^{2}\right) d s\right] \\
\leq M_{2}\left\|\psi_{0}\left(\theta_{-t} \omega\right)\right\|_{F}^{2} e^{-c_{3} t}+\frac{1}{c_{3}(\sigma+\alpha)}\|g\|^{2} e^{-c_{3}\left(t-T_{K}\right)} \\
+\frac{2 M_{3}}{2 c_{3}+\epsilon} b(\omega) e^{-(\epsilon / 2)\left(t-T_{K}\right)} .
\end{gathered}
$$

Hence, for any given constant $\mu>0$, there exists $T_{1}(\mu, \omega)>$ $T_{K}(\omega)$ such that, for all $t>T_{1}(\mu, \omega)$,

$$
\begin{aligned}
& M_{2} e^{-c_{3}\left(t-T_{K}\right)} \sum_{i \in \mathbb{Z}^{n}} \vartheta\left(\frac{\|i\|_{m}}{M}\right)\left\|\psi_{i}\left(T_{K}, \theta_{-t} \omega, \psi_{0}\left(\theta_{-t} \omega\right)\right)\right\|_{F}^{2} \\
& \quad \leq \frac{1}{4} \mu .
\end{aligned}
$$

Using (65) in Theorem 10 and property (Y3) again, we have

$$
\begin{gathered}
\int_{T_{K}}^{t} e^{c_{3}(\tau-t)}\left\|\psi\left(\tau, \omega, \psi_{0}(\omega)\right)\right\|_{F}^{2} d \tau \\
=\int_{T_{K}}^{t} e^{c_{3}(\tau-t)}\left[M_{2}\left\|\psi_{0}\left(\theta_{-t} \omega\right)\right\|_{F}^{2} e^{-c_{3} \tau}+\frac{1}{c_{3}(\sigma+\alpha)}\|g\|^{2}\right. \\
+M_{3} \int_{0}^{\tau} e^{c_{3}(s-\tau)}\left(\left\|y\left(\theta_{s-t} \omega\right)\right\|^{2 p+2}\right. \\
\left.\left.+\left\|y\left(\theta_{s-t} \omega\right)\right\|^{2}\right) d s\right] d \tau \\
\leq M_{2}\left\|\psi_{0}\left(\theta_{-t} \omega\right)\right\|_{F}^{2}\left(t-T_{K}\right) e^{c_{3} t} \\
+\frac{1}{c_{3}(\sigma+\alpha)}\|g\|^{2}+\frac{4 M_{3}}{\epsilon\left(4 c_{3}+\epsilon\right)} b(\omega) .
\end{gathered}
$$

Since $\psi_{0}\left(\theta_{-t} \omega\right) \in K\left(\theta_{-t} \omega\right)$, from Theorem 10 , we have $\left\|\psi_{0}\left(\theta_{-t} \omega\right)\right\| \leq \widetilde{B}\left(\theta_{-t} \omega\right)$. So, for any $\mu$, there exist $T_{2}(\mu, \omega)>$ $T_{K}(\omega), N_{1}(\mu, \omega)>0$, and $M>N_{1}(\mu, \omega)$ such that

$$
M_{5} \int_{T_{K}}^{t} e^{c_{3}(\tau-t)}\left\|\psi\left(\tau, \omega, \psi_{0}(\omega)\right)\right\|_{F}^{2} d \tau \leq \frac{1}{4} \mu .
$$

By assumption (C4), there exists $N_{2}(\mu, \omega)>0$ such that, for all $M>N_{2}(\mu, \omega)$, the following inequality holds:

$$
\frac{1}{c_{3}(\sigma+\alpha)} \sum_{\|i\|_{m} \geq M}\left|g_{i}\right|^{2} \leq \frac{1}{4} \mu
$$

Let $T_{0}$ be a positive constant to be determined later. When $t>T_{0}+T_{K}$, we have

$$
\begin{gathered}
M_{4} \int_{T_{K}}^{t} e^{c_{3}(s-t)} \sum_{\|i\|_{m} \geq M}\left(\left|y_{i}\left(\theta_{s-t} \omega\right)\right|^{2 p+2}+\left|y_{i}\left(\theta_{s-t} \omega\right)\right|^{2}\right) d s \\
=M_{4} \int_{T_{K}-t}^{0} e^{c_{3} \tau} \sum_{\|i\|_{m} \geq M}\left(\left|y_{i}\left(\theta_{\tau} \omega\right)\right|^{2 p+2}+\left|y_{i}\left(\theta_{\tau} \omega\right)\right|^{2}\right) d \tau \\
=M_{4} \int_{-T_{0}}^{0} e^{c_{3} \tau} \sum_{\|i\|_{m} \geq M}\left(\left|y_{i}\left(\theta_{\tau} \omega\right)\right|^{2 p+2}+\left|y_{i}\left(\theta_{\tau} \omega\right)\right|^{2}\right) d \tau \\
\quad+M_{4} \int_{T_{K}-t}^{-T_{0}} e^{c_{\mathcal{S}} \tau} \sum_{\|i\|_{m} \geq M}\left(\left|y_{i}\left(\theta_{\tau} \omega\right)\right|^{2 p+2}+\left|y_{i}\left(\theta_{\tau} \omega\right)\right|^{2}\right) d \tau
\end{gathered}
$$

Choosing $T_{0} \geq 2 /\left(2 c_{3}+\epsilon\right) \ln \left\{16 M_{4} b(\omega) / \mu\left(2 c_{3}+\epsilon\right)\right\}$ and by property $\mathrm{Y} 3$, we have

$$
\begin{aligned}
M_{4} \int_{T_{K}-t}^{-T_{0}} e^{c_{3} \tau} \sum_{\|i\|_{m} \geq M}\left(\left|y_{i}\left(\theta_{\tau} \omega\right)\right|^{2 p+2}\right. & \\
& \left.+\left|y_{i}\left(\theta_{\tau} \omega\right)\right|^{2}\right) d \tau \leq \frac{1}{8} \mu .
\end{aligned}
$$


For the fixed $T_{0}$, from Lebesgue's theorem, there exist $N_{3}(\mu, \omega)>0$ and $M>N_{3}(\mu, \omega)$ such that

$$
\begin{aligned}
M_{4} \int_{-T_{0}}^{0} e^{c_{3} \tau} \sum_{\|i\|_{m} \geq M}\left(\left|y_{i}\left(\theta_{\tau} \omega\right)\right|^{2 p+2}\right. & \\
& \left.+\left|y_{i}\left(\theta_{\tau} \omega\right)\right|^{2}\right) d \tau \leq \frac{1}{8} \mu .
\end{aligned}
$$

By setting

$$
\begin{gathered}
T(\mu, \omega)=\max \left\{T_{1}(\mu, \omega), T_{2}(\mu, \omega), T_{K}(\mu, \omega)+T_{0}(\mu, \omega)\right\}, \\
N^{*}(\mu, \omega)=\max \left\{N_{1}(\mu, \omega), N_{2}(\mu, \omega), N_{3}(\mu, \omega)\right\},
\end{gathered}
$$

then, for $t>T(\mu, \omega)$ and $M>N^{*}(\mu, \omega)$, we obtain that

$$
\begin{aligned}
& \sum_{\|i\|_{m} \geq 2 M}\left\|\psi_{i}\left(t, \theta_{-t}, \psi_{0}\left(\theta_{-t}\right)\right)\right\|_{F}^{2} \\
& \quad \leq \sum_{i \in \mathbb{Z}^{n}} \vartheta\left(\frac{\|i\|_{m}}{M}\right)\left\|\psi_{i}\left(t, \theta_{-t}, \psi_{0}\left(\theta_{-t}\right)\right)\right\|_{F}^{2} \leq \mu .
\end{aligned}
$$

It means that if constant $N(\mu, \omega)$ is large enough,

$$
\begin{aligned}
& \quad \sum_{\|i\|_{m} \geq N(\mu, \omega)}\left\|\varphi_{i}\left(t, \theta_{-t}, \psi_{0}\left(\theta_{-t}\right)\right)\right\|_{F}^{2} \\
& \quad \leq 2 \sum_{\|i\|_{m} \geq N(\mu, \omega)}\left(\left\|\psi_{i}\left(t, \theta_{-t}, \psi_{0}\left(\theta_{-t}\right)\right)\right\|_{F}^{2}+\left|y_{i}(\omega)\right|^{2}\right) \\
& \quad \leq 4 \mu
\end{aligned}
$$

holds. The proof of the lemma is now completed.

Finally, we prove the asymptotic compactness of the absorbing set $K(\omega)$.

Theorem 12. If assumptions (C1)-(C5) hold, then for any $\omega \in$ $\Lambda_{0}$ the set $K(\omega)$ is asymptotically compact.

Proof. For any $\omega \in \Lambda$, consider the sequence $d_{n}=$ $s\left(t_{n}, \theta_{-t_{n}} \omega\right) x_{n}$ in $s\left(t_{n}, \theta_{-t_{n}} \omega\right) K\left(\theta_{-t_{n}} \omega\right)$, where $x_{n} \in K\left(\theta_{-t_{n}} \omega\right)$ and $\left\{t_{n}\right\}_{n \in \mathbb{N}}$ is an increasing sequence in $\mathbb{R}^{+}$with $t_{n} \rightarrow+\infty$ as $n \rightarrow+\infty$.

First, let us show that $\left(d_{n}\right)_{n \in \mathbb{N}}$ has a convergent subsequence. Since $K(\omega)$ is a bounded absorbing set, $s\left(t_{n}, \theta_{-t_{n}} \omega\right) x_{n} \in K(\omega)$ holds for large $n$. Then, there exists a subsequence of $\left\{s\left(t_{n}, \theta_{-t_{n}} \omega\right) x_{n}\right\}$ (still denoted by $\left.\left\{s\left(t_{n}, \theta_{-t_{n}} \omega\right) x_{n}\right\}\right)$ such that

$$
s\left(t_{n}, \theta_{-t_{n}} \omega\right) x_{n} \longrightarrow x_{0} \quad \text { weakly in } F .
$$

In what follows, we prove that the weak convergence (103) is actually strong convergence. In other words, we will show that, for every $\mu>0$, there exists $\widetilde{N}(\mu, \omega)>0$ such that when $n>N_{0}(\mu, \omega)$,

$$
\left\|s\left(t_{n}, \theta_{-t_{n}} \omega\right) x_{n}-x_{0}\right\|_{F}^{2} \leq \mu .
$$

By Lemma 11 , there exist $\widetilde{N}_{1}(\mu, \omega)>0$ and $K_{1}(\mu, \omega)>0$ such that, for $n>\widetilde{N}_{1}(\mu, \omega)$,

$$
\sum_{\|i\|_{m} \geq K_{1}(\mu, \omega)}\left\|\left(s\left(t_{n}, \theta_{-t_{n}} \omega\right) x_{n}\right)_{i}\right\|_{F}^{2} \leq \frac{1}{8} \mu^{2} .
$$

Also, since $x_{0} \in F$, there exists $K_{2}(\mu)$ such that

$$
\sum_{\|i\|_{m} \geq K_{2}(\mu)}\left\|\left(x_{0}\right)_{i}\right\|_{F}^{2} \leq \frac{1}{8} \mu^{2} .
$$
that

Let $K(\mu, \omega)=\max \left\{K_{1}(\mu, \omega), K_{2}(\mu)\right\}$. By (103), we infer

$$
\begin{aligned}
& \left(\left(s\left(t_{n}, \theta_{-t_{n}}\right) x_{n}\right)_{i}\right)_{\|i\|_{m} \leq K(\mu, \omega)} \\
& \longrightarrow\left(\left(x_{0}\right)_{i}\right)_{\|i\|_{m} \leq K(\mu, \omega)} \quad \text { in } \mathbb{R}^{2 n K(\mu, \omega)+1}, \\
& \text { as } n \longrightarrow \infty,
\end{aligned}
$$

which implies that there exists $\widetilde{N}_{2}(\mu, \omega)>0$ such that when $n \geq \widetilde{N}_{2}(\mu, \omega)$,

$$
\sum_{\|i\|_{m} \leq K(\mu, \omega)}\left\|\left(s\left(t_{n}, \theta_{-t_{n}} \omega\right) x_{n}\right)_{i}-\left(x_{0}\right)_{i}\right\|_{F}^{2} \leq \frac{1}{2} \mu^{2} .
$$

Setting $\widetilde{N}(\mu, \omega)=\max \left\{\widetilde{N}_{1}(\mu, \omega), \widetilde{N}_{2}(\mu, \omega)\right\}$, we get from (105)-(108) that for $n \geq \widetilde{N}(\mu, \omega)$

$$
\begin{aligned}
\| s & \left(t_{n}, \theta_{-t_{n}} \omega\right) x_{n}-x_{0} \|_{F}^{2} \\
= & \sum_{\|i\|_{m} \leq K(\mu, \omega)}\left\|\left(s\left(t_{n}, \theta_{-t_{n}} \omega\right) x_{n}\right)_{i}-\left(x_{0}\right)_{i}\right\|_{F}^{2} \\
& +\sum_{\|i\|_{m} \geq K(\mu, \omega)}\left\|\left(s\left(t_{n}, \theta_{-t_{n}} \omega\right) x_{n}\right)_{i}-\left(x_{0}\right)_{i}\right\|_{F}^{2} \\
\leq & \frac{1}{2} \mu^{2}+\frac{1}{2} \mu^{2} \leq \mu^{2} .
\end{aligned}
$$

Therefore, we arrive at

$$
s\left(t_{n}, \theta_{t_{n}} \omega\right) x_{n} \longrightarrow x_{0} \quad \text { strongly in } F .
$$

The proof is therefore completed.

Finally, the main conclusion follows from Theorem 5, Theorem 10, and Theorem 12.

Theorem 13. Assume that (C1)-(C5) hold. Then, for any $\omega \in \Lambda$, the continuous random lattice dynamical system $s(t, \omega)$ generated by the general second-order stochastic lattice dynamical system (1) has a unique global random attractor.

\section{Conclusions}

In this paper, we have investigated the random attractors in second-order stochastic lattice dynamical systems. First, we first proved the uniqueness and existence of the solutions 
of second-order stochastic lattice dynamical systems in the space $F=l_{\lambda}^{2} \times l^{2}$. Then, by proving the asymptotic compactness of the random dynamical systems, we have established the existence of the global random attractor with the set of tempered bounded sets. These results could be further extended to more general nonlinear systems with uncertainties as in [25-27].

\section{Conflict of Interests}

The authors declare that there is no conflict of interests regarding the publication of this paper.

\section{Acknowledgments}

This work was supported in part by the National Natural Science Foundation of China under Grant nos. 61374010, 61074129, and 61175111, the Natural Science Foundation of Jiangsu Province of China under Grant BK2012682, the Qing Lan Project of Jiangsu Province (2010), the 333 Project of Jiangsu Province (2011), and the Six Talents Peak Project of Jiangsu Province (DZXX-047).

\section{References}

[1] J. Bell and C. Cosner, "Threshold behavior and propagation for nonlinear differential-difference systems motivated by modeling myelinated axons," The Quarterly of Applied Mathematics, vol. 42 , no. 1, pp. 1-14, 1984.

[2] J. W. Cahn, "Theory of crystal growth and interface motion in crystalline materials," Acta Metallurgica, vol. 8, no. 8, pp. 554$562,1960$.

[3] L. O. Chua and T. Roska, "The CNN paradigm," IEEE Transactions on Circuits and Systems I: Fundamental Theory and Applications, vol. 40, no. 3, pp. 147-156, 1993.

[4] T. L. Carroll and L. M. Pecora, "Synchronization in chaotic systems," Physical Review Letters, vol. 64, no. 8, pp. 821-824, 1990.

[5] S.-N. Chow and J. Mallet-Paret, "Pattern formation and spatial chaos in lattice dynamical systems. I," IEEE Transactions on Circuits and Systems. I. Fundamental Theory and Applications, vol. 42, no. 10, pp. 746-751, 1995.

[6] Y. R. Liu, Z. R. Liu, and Y. A. Zheng, "Attractors of nonautonomous Schrödinger equations," Applied Mathematics and Mechanics, vol. 22, no. 2, pp. 180-189, 2001.

[7] W. J. Firth, “Optical memory and spatial chaos," Physical Review Letters, vol. 61, no. 3, pp. 329-332, 1988.

[8] J. P. Keener, "Propagation and its failure in coupled systems of discrete excitable cells," SIAM Journal on Applied Mathematics, vol. 47 , no. 3, pp. 556-572, 1987.

[9] S.-N. Chow, J. Mallet-Paret, and W. Shen, "Traveling waves in lattice dynamical systems," Journal of Differential Equations, vol. 149, no. 2, pp. 248-291, 1998.

[10] J. K. Hale, Asymptotic Behavior of Dissipative Systems, American Mathematical Society, Providence, RI, USA, 1988.

[11] R. Temam, Infinite-Dimensional Dynamical Systems in Mechanics and Physics, vol. 68 of Applied Mathematical Sciences, Springer, New York, NY, USA, 2nd edition, 1997.
[12] P. W. Bates, K. Lu, and B. Wang, "Attractors for lattice dynamical systems," International Journal of Bifurcation and Chaos in Applied Sciences and Engineering, vol. 11, no. 1, pp. 143-153, 2001.

[13] S. F. Zhou, "Attractors for second order lattice dynamical systems with damping," Journal of Mathematical Physics, vol. 43, no. 1, pp. 452-465, 2002.

[14] D. Ruelle, "Characteristic exponents for a viscous fluid subjected to time dependent forces," Communications in Mathematical Physics, vol. 93, no. 3, pp. 285-300, 1984.

[15] H. Crauel, A. Debussche, and F. Flandoli, "Random attractors," Journal of Dynamics and Differential Equations, vol. 9, no. 2, pp. 307-341, 1997.

[16] H. Crauel and F. Flandoli, "Attractors for random dynamical systems," Probability Theory and Related Fields, vol. 100, no. 3, pp. 365-393, 1994.

[17] F. Flandoli and B. Schmalfuss, "Random attractors for the 3D stochastic Navier-Stokes equation with multiplicative white noise," Stochastics and Stochastics Reports, vol. 59, no. 1-2, pp. 21-45, 1996.

[18] P. Imkeller and B. Schmalfuss, "The conjugacy of stochastic and random differential equations and the existence of global attractors," Journal of Dynamics and Differential Equations, vol. 13, no. 2, pp. 215-249, 2001.

[19] P. W. Bates, H. Lisei, and K. N. Lu, "Attractors for stochastic lattice dynamical systems," Stochastics and Dynamics, vol. 6, no. 1, pp. 626-637, 2006.

[20] Y. Lv and J. Sun, "Dynamical behavior for stochastic lattice systems," Chaos, Solitons and Fractals, vol. 27, no. 4, pp. 10801090, 2006.

[21] A. Gu, "Random attractors of stochastic lattice dynamical systems driven by fractional Brownian motions," International Journal of Bifurcation and Chaos in Applied Sciences and Engineering, vol. 23, no. 3, Article ID 1350041, 2013.

[22] X. Wang, S. Li, and D. Xu, "Random attractors for secondorder stochastic lattice dynamical systems," Nonlinear Analysis: Theory, Methods \& Applications, vol. 72, no. 1, pp. 483-494, 2010.

[23] S. Zhou and L. Wei, "A random attractor for a stochastic second order lattice system with random coupled coefficients," Journal of Mathematical Analysis and Applications, vol. 395, no. 1, pp. 42-55, 2012.

[24] L. Arnold, Random Dynamical Systems, Springer, Berlin , Germany, 1998.

[25] M. Kermani and A. Sakly, "Stability analysis for a class of switched nonlinear time-delay systems," Systems Science and Control Engineering, vol. 2, pp. 80-89, 2014.

[26] A. J. Shaiju and I. R. Petersen, "Discrete-time robust $H_{\infty}$ control of a class of nonlinear uncertain systems," International Journal of Robust and Nonlinear Control, vol. 23, no. 14, pp. 1629-1641, 2013.

[27] S. Zhang, Z. Wang, D. Ding, and H. Shu, " $H_{\infty}$ fuzzy control with randomly occurring infinite distributed delays and channel fadings," IEEE Transactions on Fuzzy Systems, vol. 22, no. 1, pp. 189-200, 2014 


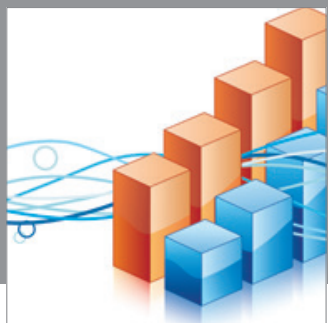

Advances in

Operations Research

mansans

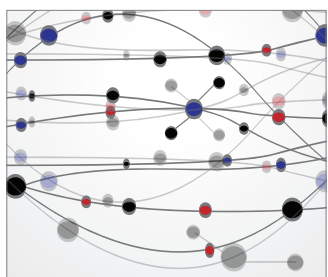

The Scientific World Journal
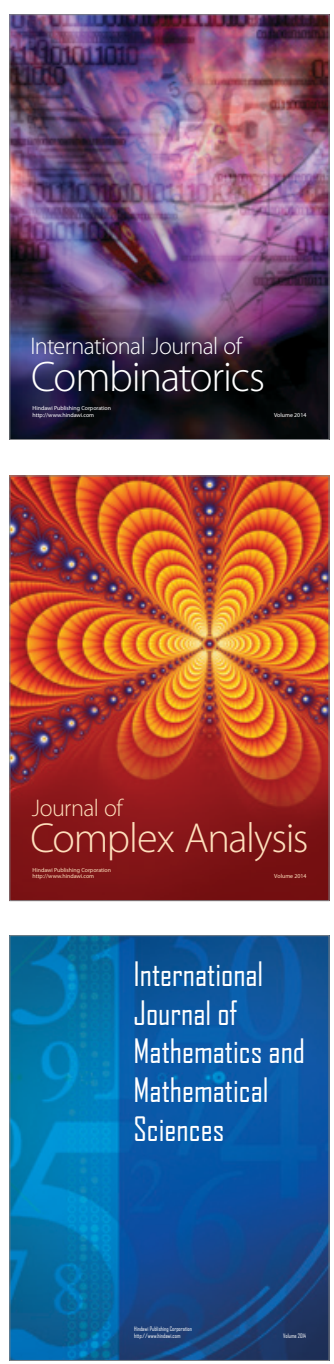
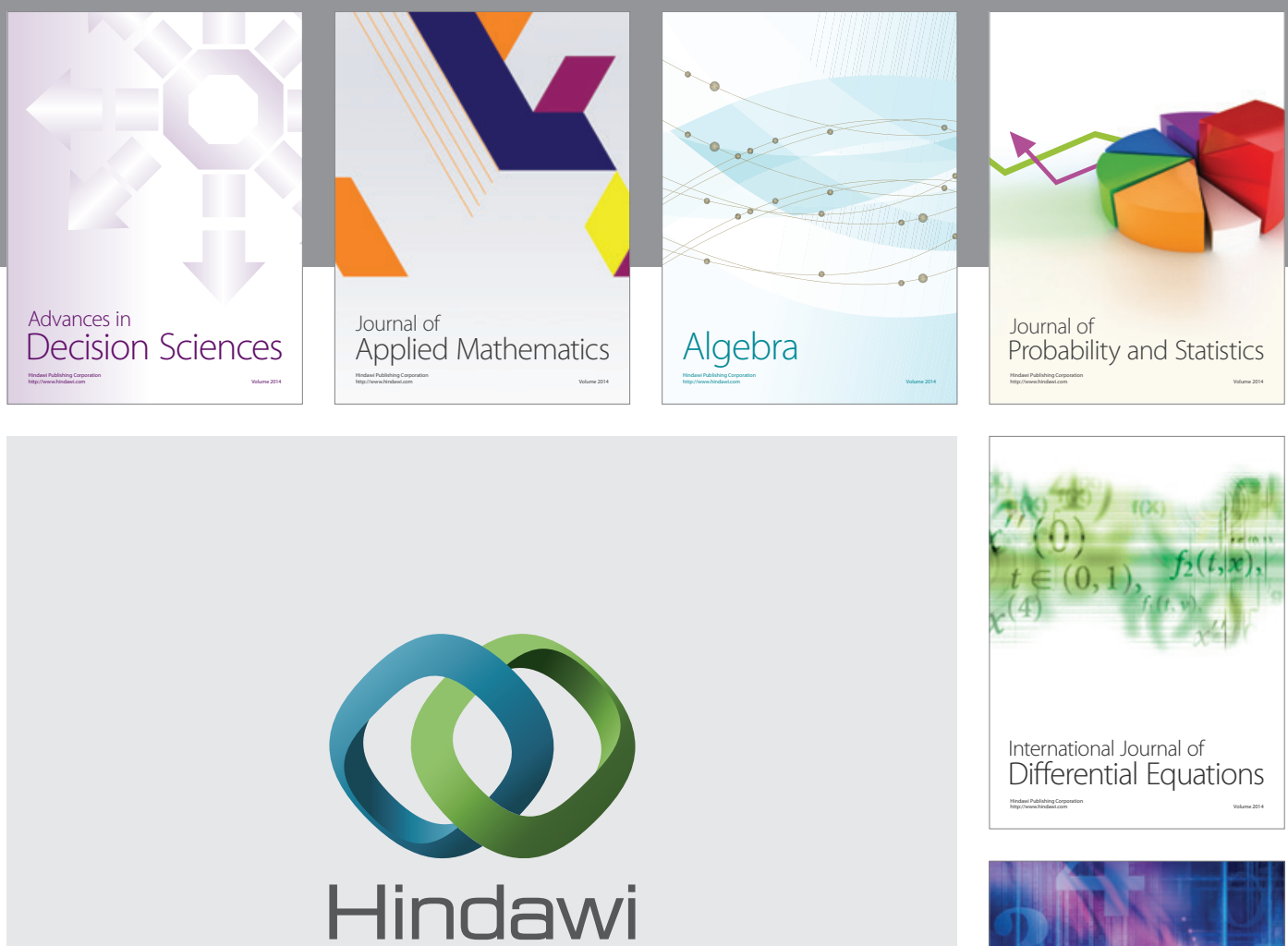

Submit your manuscripts at http://www.hindawi.com
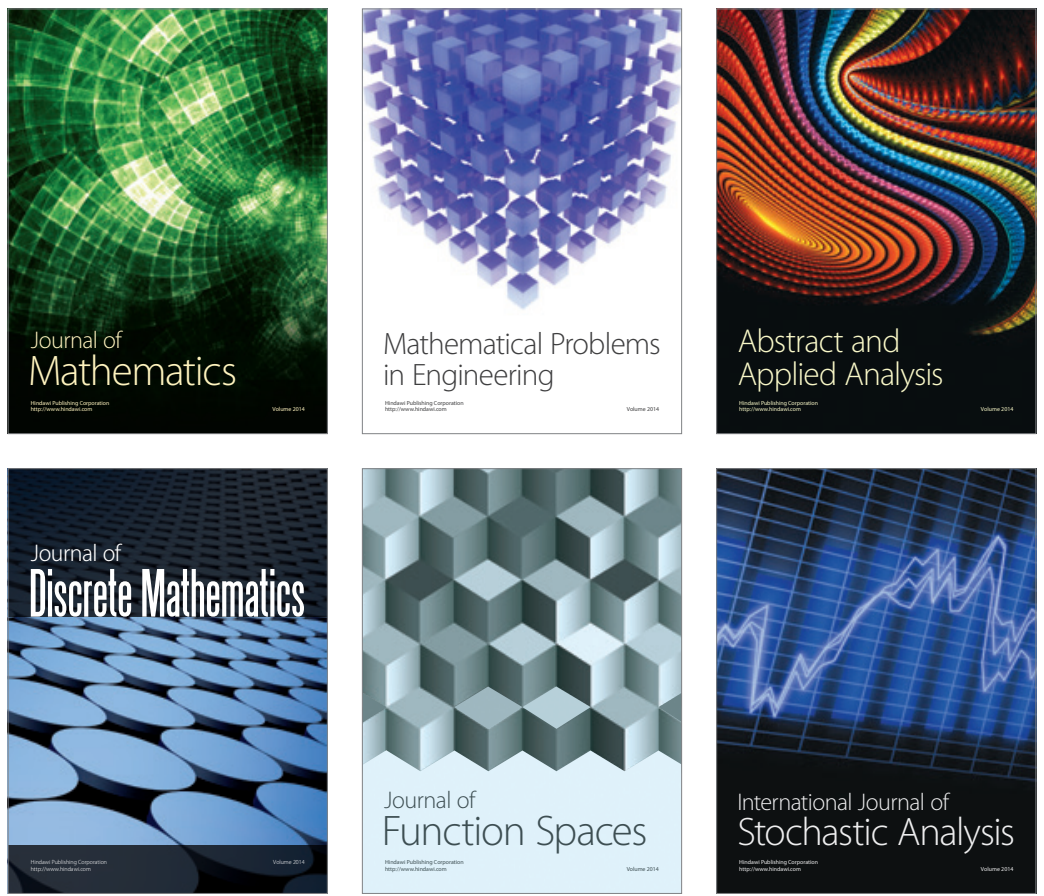

Journal of

Function Spaces

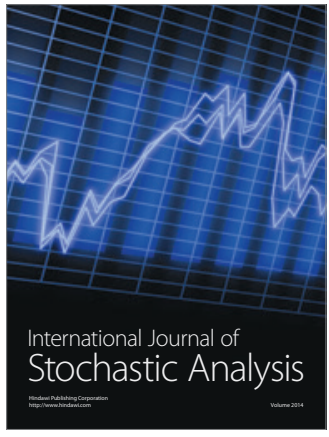

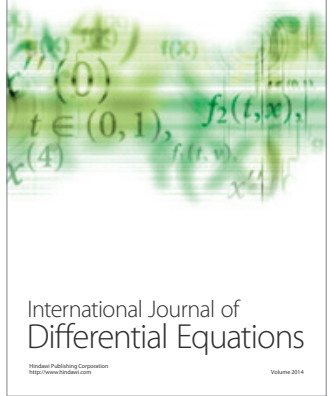
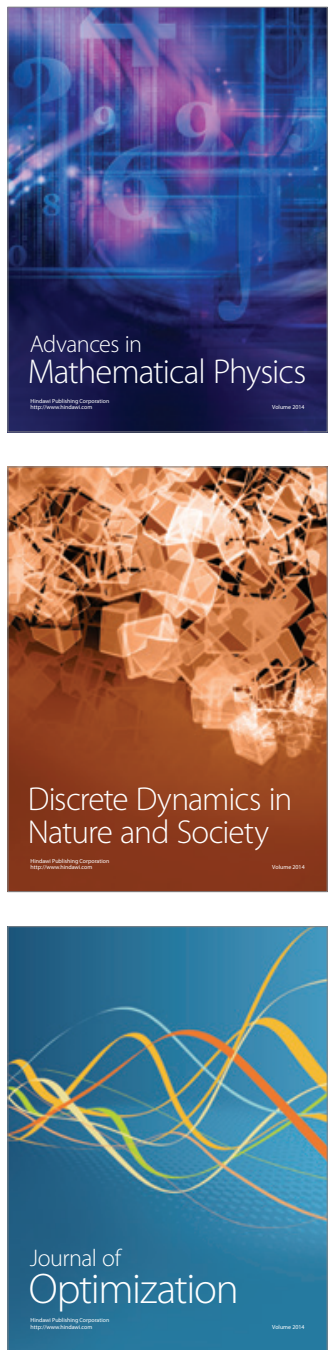